\title{
Potent Natural Antioxidant Carveol Attenuates MCAO-Stress Induced Oxidative, Neurodegeneration by Regulating the Nrf-2 Pathway
}

\author{
Imran Malik ${ }^{1,2}$, Fawad Ali Shah ${ }^{1 *}$, Tahir Ali ${ }^{3}$, Zhen Tan ${ }^{4 *}$, Abdullah Alattar, \\ Najeeb Ullah', ${ }^{2,6}$, Arif-ullah Khan ${ }^{1}$, Reem Alshaman ${ }^{5}$ and Shupeng Li ${ }^{2 *}$ \\ ${ }^{1}$ Riphah Institute of Pharmaceutical Sciences, Riphah International University, Islamabad, Pakistan, ${ }^{2}$ State Key Laboratory of \\ Oncogenomics, School of Chemical Biology and Biotechnology, Shenzhen Graduate School, Peking University, Shenzhen, \\ China, ${ }^{3}$ Department of Comparative Biology and Experimental Medicine, Faculty of Veterinary Medicine, Hotchkiss Brain \\ Institute, Cumming School of Medicine, University of Calgary, Calgary, AB, Canada, ${ }^{4}$ Health Management Center, Shenzhen \\ University General Hospital, Shenzhen University Clinical Medical Academy, Shenzhen, China, ${ }^{5}$ Department of \\ Pharmacology and Toxicology, Faculty of Pharmacy, University of Tabuk, Tabuk, Saudi Arabia, ${ }^{6}$ Institute of Basic Medical \\ Sciences, Khyber Medical University, Peshawar, Pakistan
}

OPEN ACCESS

Edited by:

Anna Karolina Kiss,

Medical University of Warsaw, Poland

Reviewed by:

Ying $X u$,

University at Buffalo, United States

Wladyslaw-Lason,

Maj Institute of Pharmacology (IF

PAS), Poland

*Correspondence:

Fawad Ali Shah

fawad.shah@riphah.edu.pk

Zhen Tan

tanzhen@szu.edu.cn

Shupeng Li

lisp@pku.edu.cn

Specialty section:

This article was submitted to

Neuropharmacology,

a section of the journal

Frontiers in Neuroscience

Received: 17 March 2020

Accepted: 28 May 2020

Published: 26 June 2020

Citation:

Malik I, Shah FA, Ali T, Tan Z,

Alattar A, Ullah N, Khan A, Alshaman R and Li S (2020) Potent

Natural Antioxidant Carveol Attenuates MCAO-Stress Induced Oxidative, Neurodegeneration by

Regulating the Nrf-2 Pathway.

Front. Neurosci. 14:659

doi: 10.3389/fnins.2020.00659
Ischemic stroke is a severe neurological disorder with a high prevalence rate in developed countries. It is characterized by permanent or transient cerebral ischemia and it activates syndrome of pathological events such as membrane depolarization, glutamate excitotoxicity, and intracellular calcium buildup. Carveol is widely employed as anti-inflammatory and antioxidant in traditional Chinese medicine. In the present study, the neuroprotective effects of post-treated carveol were demonstrated against transient middle cerebral artery occlusion (MCAO) induced focal ischemic cerebral injury. Male Sprague Dawley (SD) rats were subjected to two different experimental protocols to determine the dose and effects of carveol, and to demonstrate the underlying role of the nuclear factor E2-related factor (Nrf2) pathway. Our results showed that MCAO induced marked neuronal injury in the ipsilateral cortex and striatum associated with higher inflammatory cytokines expression, along with apoptotic markers such as caspase-3 and the phosphorylated c-Jun N-terminal kinase (JNK). Furthermore, MCAO induced a marked increase in oxidative stress as evidenced by high lipid peroxidase (LPO) content accompanied by the depressed antioxidant system. Carveol significantly reversed the oxidative stress and downregulated inflammatory cascades by enhancing endogenous antioxidant mechanisms including the Nrf2 gene, which critically regulates the expression of several downstream antioxidants. Further, to determine the possible involvement of Nrf2 in carveol mediated neuroprotection, we antagonized Nrf2 by alltrans retinoic acid (ATRA), and such treatment abrogated the protective effects of carveol accompanied with exaggerated neuronal toxicity as demonstrated by higher infarction area. The target effects of carveol were further supported by molecular docking analysis of drug-protein interactions. Together, our findings suggest that carveol could activate endogenous master anti-oxidant Nrf2, which further regulates the expression of downstream antioxidants, eventually ameliorating MCAO-induced neuroinflammation and neurodegeneration.

Keywords: middle cerebral artery occlusion, carveol, all-trans retinoic acid, antioxidant enzymes, Nrf2 pathway 


\section{INTRODUCTION}

Ischemic stroke is a neurological disorder with a high prevalence in developed countries. Ischemic stroke has a higher incidence ratio of about (80\%) as compared to other types and occurs due to occlusion in the middle cerebral artery (MCA) (Dirnagl et al., 1999). In ischemic stroke, either permanent or briefly transient hindrance of blood flow occurs which leads to decreased oxygen and nutrient supply to the brain. Tissue plasminogen activator (tPA) is the only FDA approved drug for stroke which acts via vascular recanalization. Ischemic injury can cause a varying degree of cellular damage marked by complex events of signaling cascades (Dirnagl et al., 1999). Immediately after the stroke, ATP and energy production are reduced along with ionic imbalance, which sequentially leads to membrane depolarization and calcium overload. Furthermore, excessive release of glutamate enhances the influx of calcium ions and triggers downstream inflammatory and apoptotic proteins (Barone and Feuerstein, 1999; Wang Q. et al., 2007) along with rapid activation of glia cells (Chung and Benveniste, 1990). This, mingled with the vascular and tissue damage incurred by early mitochondrial dysfunction and oxidative phosphorylation, trigger the release of reactive oxygen species (ROS) and pro-inflammatory mediators such as interleukin-1 (IL-1 $\beta$ ), tumor necrosis factor-alpha (TNF$\alpha$ ), and interleukin-6 (IL-6), eventually leading to cellular damage as well as peroxidation of membranous protein and cytoplasmic organelles (Vila et al., 2000). Neuro-inflammation remains the more prominent pathological hallmark of ischemic stroke as it starts hours after ischemia and disseminates to other biochemical cascades for secondary damages. Combating neuro-inflammation is, therefore, an attractive strategy to prevent MCA occlusion (MCAO) induced degeneration. Several lines of evidence and comprehensive literature review enlighted that activation of endogenous and master anti-oxidant Nrf2 (nuclear factor E2-related factor or nuclear factor erythroid 2 or p45-related factor 2) plays an important role in the impaired cellular homeostasis via responding to endogenous and exogenous stressors/insults in different diseases (Suzuki and Yamamoto, 2015; Javeed et al., 2017; Vomund et al., 2017; Liu et al., 2019). Consistent reports reiterated the protective role of Nrf2 in the brain and metabolic disorders (Ramsey et al., 2007; Dinkova-Kostova et al., 2018; Sivandzade et al., 2019). Nrf2 is ubiquitously present in the cytoplasm as a dimer with Keap1 (Nrf2-Keap1), whereas Keap1 inhibits or suppresses Nrf2 activation. Upon activation by stress or exposure to ROS, Nrf2 translocates to the nucleus to activate antioxidant machinery and negatively regulates downstream pro-inflammatory mediators such as $\mathrm{NF}_{K} \mathrm{~B}$, and inflammatory cytokines (TNF- $\alpha$ and COX2) (Lee and Johnson, 2004; Hennig et al., 2018). Most importantly, Nrf2 activation and its associated signalings are neuroprotective in ischemic and other brain injuries in various pre-clinical animal models (Hong et al., 2010; Jiang et al., 2017; Zhang et al., 2018; Duan et al., 2019; Shah et al., 2019a). Thus, Nrf2 dependent signaling pathway presents an appropriate therapeutic target to cope against a variety of insults including cerebral ischemia.

Natural products have been exploited as a repository for novel therapeutic identification for decades and thus attracted considerable attention as a source of potential therapeutic agents. This can be attributed to its antioxidant nature consistently validated in experimental models by many researchers. Our group is also aimed to explore the natural-based compounds to prevent and treat ischemic associated detrimental effects (Sung et al., 2012; Gim et al., 2013; Shah et al., 2014, 2019a). Herein, we hypothesized that natural derived substance, carveol act as potent anti-oxidant and prevent MCAO induced brain degeneration. Carveol, a natural monocyclic monoterpenoid abundantly present in the essential oil of orange peel, dill, and caraway seeds (De Carvalho and Da Fonseca, 2006). It is found in caraway, mandarin (Citrus reticulata), blackcurrant berries, black tea, and dill (Crowell et al., 1992). The caraway based medicinal plants have been useful in the management of various diseases (Mahboubi, 2019). Carveol has been reported in traditional Chinese medicine as an antispasmodic, carminative, astringent, and further use/used for indigestion and dyspepsia (Sachan et al., 2016). It also demonstrated antioxidant, anti-hyperlipidemic, and anti-inflammatory activities in the liver (Johri, 2011). Furthermore, carveol is equally potent to inhibit COX2 activation and its anti-inflammatory effects are comparable to that of aspirin (Kawata et al., 2008). Nevertheless, as per our knowledge and literature survey, there is no study on carveol in neurodegenerative diseases and ischemic associated brain degeneration. The present study aims to evaluate whether carveol effects on neuroinflammation and oxidative stress could eventually account for cellular protection. If so, the potential molecular and cellular mechanisms underlying these effects need to be further delineated. Results obtained will not only help us to understand the relationship of cascading mechanisms that eventually lead to cell death, but also provide a clue as to the potentials of Nrf2 targeting therapeutics.

\section{MATERIALS AND METHODS}

\section{Chemicals and Reagents}

Mouse monoclonal anti-HO1 (SC-136960), rabbit polyclonal anti Nrf2 (SC-722), mouse monoclonal anti-p-JNK (SC6254), mouse monoclonal anti-COX-2 (SC-514489), mouse monoclonal anti-p-NFKB (SC-271908), mouse monoclonal anti-Bcl2 (SC-7382), mouse monoclonal anti-caspase-3 (SC56053), ABC Elite kit (SC-2018), Ultra Cruz mounting media (SC-516212), mouse anti-rabbit IgG-R (SC-2492), and 3,3'-diaminobenzidine peroxidase (DAB) (SC-216567) were purchased from Santa Cruz Biotechnology. The horseradish peroxidase-conjugated secondary antibodies were obtained from Abcam (ab-6789, ab-6721). p-NF-kB Elisa kit (Cat \# SUB28069) and Nrf2 Elisa kit (cat. no. SU-B30429) were purchased from (Shanghai Yuchun Biotechnology, China). HO-1 Elisa kit (cat. No. E-EL-R0488), and TNF- $\alpha$ Elisa kit (cat. No. E-EL-R0019) were got from Elabscience. PBS tablets and proteinase $\mathrm{K}$ were obtained from (MP Bio, United States). Formaldehyde, hydrogen peroxide $\left(\mathrm{H}_{2} \mathrm{O}_{2}\right)$, reduced glutathione (GSH), trichloroacetic acid (TCA), 1-chlor-2,4-dinitrobenzene (CDNP), $N$-(1-naphthyl)ethylenediamine dihydrochloride, 5,5'dithio-bis-(2-nitrobenzoic acid) (DTNB), and carveol (catalog 
No: 192384 a mixture of isomers, with $97 \%$ purity) was purchased from (Sigma-Aldrich, United States). All-trans retinoic acid (ATRA) of the highest analytical grade (99\% HPLC) was purchased from the local pharmaceutical industry (GSK).

\section{Animal Grouping and Drug Treatment}

Adult male Sprague-Dawley rats weighing 230-260 g, 710 weeks were obtained from Riphah International University, Islamabad. The experimental animals were housed at Laboratory Animal Research Center, Riphah International University, under $12 \mathrm{~h}$ light $/ 2 \mathrm{~h}$ dark cycle at $18-22^{\circ} \mathrm{C}$ and had free access to diet and tap water throughout the study. The experimental procedures were set in such a way to minimize rats suffering. All experimental procedures were carried out according to the protocols approved by the Institutional Animal Care and Use Committee of Riphah Institute of Pharmaceutical Sciences (ref no.: REC/RIPS/2018/06) and were strictly adhered to the approved protocols, in addition, to ARRIVE guidelines with few exceptions. We did not apply human endpoints for euthanizing the rats as the ischemic stroke (MCAO model) is the most stressful invasive procedure and in which limited mobility with severe suffering is an established documented protocol, and our group was more interested in rats that survive this period. The exclusion criteria include animals showing no depressed signs or alteration in movements after awakening from anesthesia. By this, we did not euthanize any rats until $72 \mathrm{~h}$ of the ischemic period. We applied all laboratory procedures to minimize rat sufferings such as heating pad, sterilization, and fluid replenishment with normal saline. The rats were randomly divided into the following six groups ( $n=17 /$ group, Figure 1):

1. Vehicle treated control group/Sham; rats in this group received normal saline (containing 5\% DMSO).

2. Transient MCAO (t-MCAO) group. MCAO was carried out for $120 \mathrm{~min}$ followed by $72 \mathrm{~h}$ reperfusion.

3. $\mathrm{CR}+\mathrm{MCAO}$ group: carveol dissolved in a mixture of normal saline and 5\% DMSO and was administrated at $30 \mathrm{~min}, 24,48$, and $72 \mathrm{~h}$ after the MCAO (at doses of 10 and $20 \mathrm{mg} / \mathrm{kg}$ ).

4. ATRA + MCAO group: single-dose $(5 \mathrm{mg} / \mathrm{kg})$ of ATRA dissolved in normal saline (containing 5\% DMSO) was administered intraperitoneally $30 \mathrm{~min}$ before the induction of ischemia.

5. CR + ATRA + MCAO group: ATRA was administrated as mentioned and carveol $(20 \mathrm{mg} / \mathrm{kg})$ was administered intraperitoneally $30 \mathrm{~min}, 24,48$, and $72 \mathrm{~h}$ after ischemia.

All rats were sacrificed $6 \mathrm{~h}$ after the last treatment. A total of 10 animals died during the experimental procedures including three from the MCAO group, three from low dose carveol (10 mg/kg), one from high dose carveol $(20 \mathrm{mg} / \mathrm{kg})$, two from $\mathrm{CR}+\mathrm{ATRA}+\mathrm{MCAO}$ group and one from the sham group, which we further adjusted by supplementing more animals. The reported reason for this mortality is edema formation, BBB leakage, and hypothalamic shutdown (Neumann-Haefelin et al., 2000). The ethics committee is mostly aware of the mortality in experimental setup particularly in this model, as we constantly engaged them for our work.

\section{The MCAO Surgery}

The animals were anesthetized with an I/P administration of a cocktail of xylazine $(9 \mathrm{mg} / \mathrm{kg})$ and ketamine $(91 \mathrm{mg} / \mathrm{kg})$. The body core temperature was maintained using a heating pad. MCAO was carried out as previously described (Ali et al., 2020; Park et al., 2020; Shah et al., 2016). Briefly, the right common carotid artery (R-CCA) and its bifurcating branches; internal and external carotid arteries were exposed after a midline cervical incision. Superior thyroid artery and the occipital artery, small protrusions from the external carotid artery were identified and knotted with a thin black (6/0) silk and eventually ligated to allow free movement of the external carotid artery. The external common carotid artery was then tied with silk $(6 / 0)$ near the hyoid bone above the ligated superior thyroid artery and immediately incised near the bifurcating point. Throughout this procedure, extra care was exercised to avoid excessive bleeding. A blue nylon filament (3/0) with a blunted round end was inserted through the pierced opening of the external carotid artery and advanced into the internal carotid artery up to 18$19 \mathrm{~mm}$ (depending upon the weight and age of the rat) till the origin of the MCA, whereas a small resistance to the advancement of nylon indicated occlusion of MCA. The nylon is then tied in place with the lumen of the external carotid artery, and the skin was then sealed. All animals were subjected to carbon dioxide $\left(\mathrm{CO}_{2}\right)$ euthanasia for sample collection. The sham group was exposed to similar measures but with no nylon insertion. The only lacking in this method is the absence of the Doppler effect and relative blood flow measurement, though we constantly do the occlusion with suitable experimental skills. The exclusion criteria include animals showing no depressed signs or alteration in movements after awakening from anesthesia. No significant adverse effects were observed in drug-treated animals as pilot toxicity studies indicated a significantly greater dose.

\section{Neurobehavioral Test}

The rats were handled gently and adequately trained at least 3 days before the experimentation. The behavioral studies were conducted at 24,48 , and $72 \mathrm{~h}$ post-surgery. For the determination of sensorimotor function, modified 28-point neuro-scoring was conducted (Shah et al., 2019b). The modified 28-point neuroscoring includes 11 tests having accumulative of 28 score: (1) circling (maximum four points), (2) motility (maximum three points), (3) general condition (maximum three points), (4) righting reflex when placed on the back (maximum one point), (5) paw placement of each paw onto a tabletop (maximum four points), (6) ability to pull self-up on a horizontal bar (maximum three points), (7) climbing on an inclined platform (maximum three points), (8) grip strength (maximum two points), (9) contralateral reflex (maximum one point), (10) contralateral rotation when held by the base of the tail (maximum two points), and (11) visual forepaw reaching (maximum two points). The score ranges from 0 to 28,0 indicating severe impairment, and 28 for no neurological damage. 


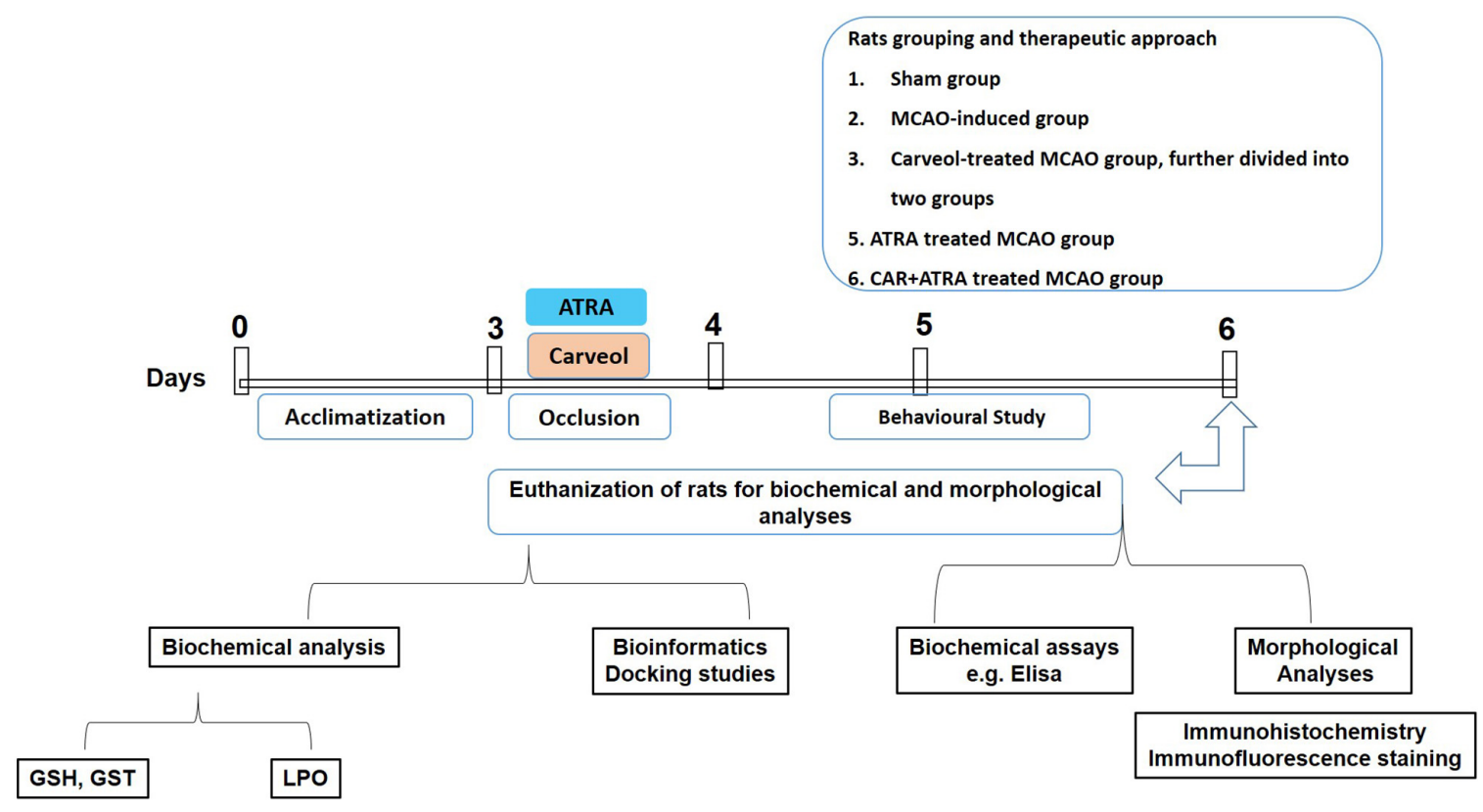

FIGURE 1 | Schematic representation of the in vivo study design. Rats acclimatization, MCAO surgery, drug administration, neurobehavioral studies, euthanization of rats for tissue collection to carry out biochemical analysis, i.e., GST, GSH, catalase and lipid peroxidation (LPO), morphological analysis (2,3,4-triphenyl tetrazolium chloride staining, hematoxylin, and eosin staining and immunohistochemistry), and biochemical assay (enzyme-linked immunosorbent assay, ELISA).
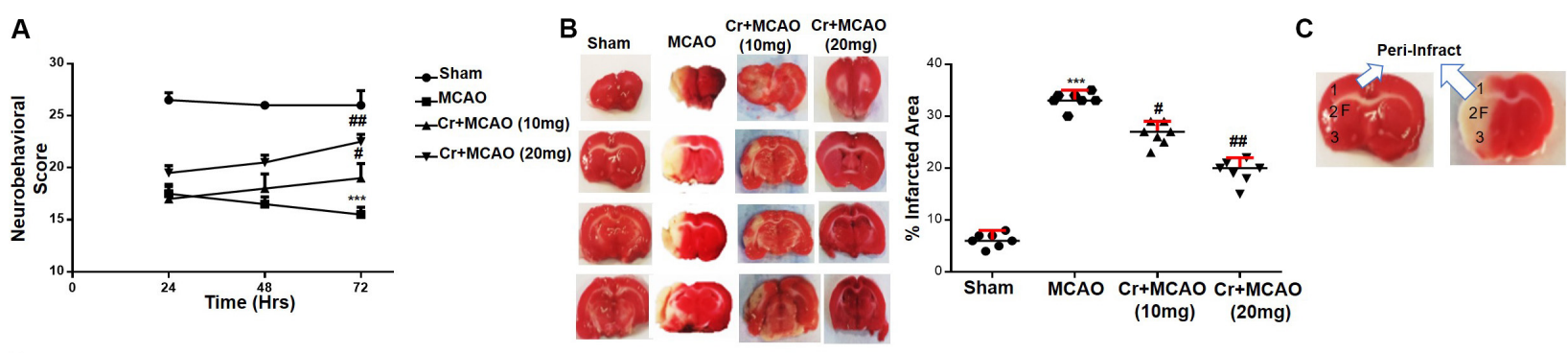

D
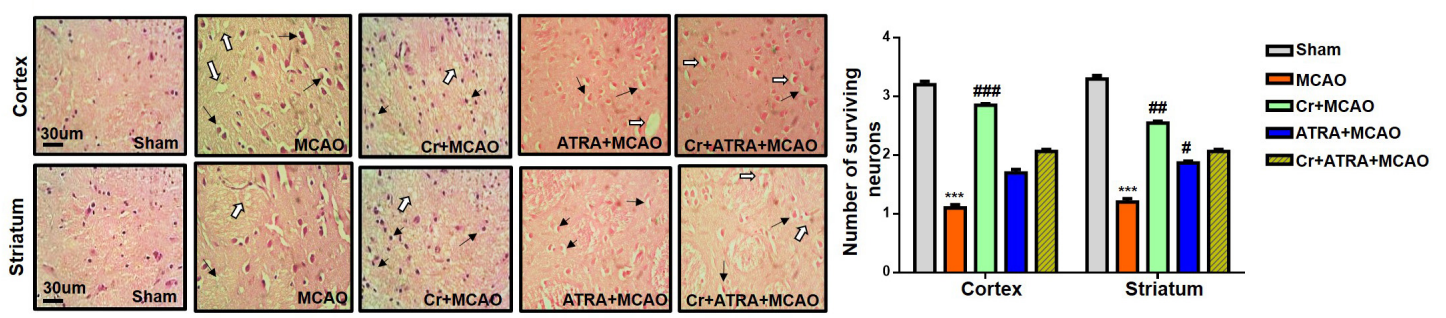

\section{H.E Staining}

FIGURE 2 | Effect of post-treatment dosage regimen of carveol on brain infarction and neuronal cell loss. (A) 28 points composite scoring. CR + MCAO rats had significantly reduced neurological deficits ( $\left.\#<0.05 \mathrm{vs}{ }^{\# \#} p<0.01\right)$ as compared to MCAO rats. Data are presented as mean \pm SEM and analyzed by two-way ANOVA (group analysis) ( $n=7 /$ group). (B) Brain coronal sections stained with TTC were used for analysis. Data are presented as mean \pm SEM and analyzed by one-way ANOVA ( $n=7 /$ group). ${ }^{* * *} p<0.001,{ }^{\#} p<0.05$, and ${ }^{\# \#} p<0.01$ significantly different. The symbol ${ }^{*}$ shows significant difference relative to sham while \# shows significant difference relative to MCAO. (C) Coronal sections separated by frontal cortex (1), parietal cortex and insular cortex (2), and the piriform cortex (3). The analyzed region of interests indicated by 1 and F. (D) Representative images of hematoxylin and eosin staining in various groups ( $n=5 /$ group), carveol used as $20 \mathrm{mg} / \mathrm{kg}$ and ATRA as $5 \mathrm{mg} / \mathrm{kg}$. H.E slides were made by processing TTC thick coronal sections which were fixed in $4 \%$ paraformaldehyde. From the thick coronal TTC sections, paraffin blocks were made, and later $4 \mu \mathrm{m}$ thin coronal sections were prepared by a rotary microtome. Arrows indicate shrunken and condensed nuclei and open arrows indicate swelled and vacuolated forms. Scale bar $=50 \mu \mathrm{m}$. * Significant difference relative to control; \# significant difference relative to MCAO group. All data are presented as means \pm SEM. Data were analyzed using two-way ANOVA followed by post hoc Bonferroni multiple comparison test using GraphPad Prism 6 software. ${ }^{* \star *}$ Indicates $p<0.001$ shows significant difference relative to sham control while, \#\# indicates $p<0.01$ and $\#$ indicates $p<0.05$ showing significant difference relative to MCAO group. 


\section{Brain Water Content}

Brain edema was quantitated as described previously. Briefly, animals were decapitated under anesthesia and brain samples were removed. The whole-brain was immediately weighed on an electronic analytical balance to obtain the wet weight. Brain samples were then dried at $120^{\circ} \mathrm{C}$ for $6-8 \mathrm{~h}$ to obtain the dry weight. The formula used was as follows:

(wet weight - dry weight)/wet weight $\times 100$.

\section{Staining and Histological Preparation}

After being evaluated for neurological deficits, rats were decapitated under anesthesia, and brain tissues were carefully removed. A total of 2-mm thick coronary sections were cut from the frontal lobe with a sharp blade. Slices were incubated in $2 \%$ 2,3,5-triphenyl tetrazolium chloride (TTC) for 10 to $20 \mathrm{~min}$ in a water bath at $37^{\circ} \mathrm{C}$ until a thorough demarcation was observed, and then fixed in $4 \%$ paraformaldehyde solution and photographed (Sung et al., 2016; Shah et al., 2018). The infarct area was then measured with the ImageJ program and expressed as a percentage to the total area.

To compensate for brain edema, the percent corrected brain infarction was calculated as follows:

\section{Corrected infarct area $=[$ left hemisphere area -}

(right hemisphere area - infarctarea)]/100

After photographing, these thick coronal sections were embedded in paraffin, and $4-\mu \mathrm{m}$ thin coronary sections were made by using a rotary microtome. The slides were deparaffinized in xylene and rehydrated in graded alcohol and proceeded to the following staining techniques.

\section{Hematoxylin Eosin (H\&E) Staining}

Tissue sections on coated slides were de-paraffinized with absolute xylene (100\%), rehydrated with ethyl alcohol (from 100 to $70 \%$ ). The slides were rinsed with distilled water and immersed in hematoxylin for $10 \mathrm{~min}$. After due time, the slides were traced for nuclear staining, and if the staining were not satisfactory, the hematoxylin time was further increased. The slides were then kept under running water in a glass jar for $10 \mathrm{~min}$ and treated with $1 \% \mathrm{HCL}$ and $1 \%$ ammonia water as previously reported (Gim et al., 2015). The slides were added to the eosin solution for 5-10 min. Slides were then rinsed in water and air-dried for some time. The dried slides were dehydrated in graded ethyl alcohol (70, 95, and 100\%). The slides were cleared with xylene and were mounted with a glass coverslip. The slides were pictured with a light microscope (Olympus, Japan) and analyzed by ImageJ, a computer-based program. The number of images per slide was five per group while focusing specifically on vacuoles formation, the number of surviving neurons, and infiltrations (Shah et al., 2018). The TIFF images were optimized to the same threshold intensity for all groups (Sung et al., 2016).

\section{Immunohistochemical Analysis}

Immunohistochemical staining was performed as previously described with minor modifications (Shah et al., 2019c; Iqbal et al., 2020). The slides were processed for the antigen retrieval step (enzymatic method), then washed with PBS. The endogenous peroxidase was quenched by applying $3 \%$ hydrogen peroxide $\left(\mathrm{H}_{2} \mathrm{O}_{2}\right)$ in methanol for $10 \mathrm{~min}$. The slides were incubated with $5 \%$ normal goat serum containing $0.1 \%$ Triton $\mathrm{X}-100$. After being blocked, the slides were incubated overnight in mouse anti-phosphorylated-c-Jun $N$-terminal kinase (pJNK), mouse monoclonal anti-(COX-2), mouse monoclonal anti (HO-1), mouse monoclonal anti (Nrf2), mouse antip-nuclear factor- $\kappa \mathrm{B}(\mathrm{NF}-\kappa \mathrm{B})$, mouse monoclonal anti-Bcl2, mouse monoclonal anti-caspase-3 (dilution 1:100, Santa Cruz Biotechnology, United States). The following morning, after being washed with $0.1-\mathrm{M}$ PBS, the slides were incubated in biotinylated secondary antibodies (dilution 1:50) according to the origin of the primary antibody and serum used, then incubated with $\mathrm{ABC}$ reagents (SCBT, United States) for $1 \mathrm{~h}$ in a humidified chamber. The slides were washed with 0.1-M PBS, stained in DAB solution, washed with distilled water, dehydrated in a graded ethanol series, fixed in xylene and cover-slipped in mounting medium. ImageJ software was used to quantitatively determine hyperactivated p-JNK, COX-2, HO-1, Nrf2, p-NFKB, Bcl2, and caspase 3 in cortex/total area and in the striatum/total area by optimizing background of images according to the threshold

TABLE 1 | Carveol $(20 \mathrm{mg} / \mathrm{kg})$ ameliorated oxidative stress and neuroinflammation.

\begin{tabular}{|c|c|c|c|c|}
\hline Groups & $\begin{array}{l}\text { GSH ( } \mu \text { moles } / \mathrm{mg} \text { of } \\
\text { protein) }\end{array}$ & $\begin{array}{l}\text { GST ( } \mu \text { moles CDNB conjugate/ } \\
\mathrm{min} / \mathrm{mg} \text { of protein) }\end{array}$ & $\begin{array}{l}\text { Catalase ( } \mu \text { moles } \\
\mathrm{H} 202 / \mathrm{min} / \mathrm{mg} \text { of protein) }\end{array}$ & $\begin{array}{l}\text { LPO(Tbras-nM/min } / \mathrm{mg} \\
\text { protein) }\end{array}$ \\
\hline Sham & $60.7 \pm 0.84$ & $43.2 \pm 1.62$ & $25.0 \pm 2.61$ & $40.5 \pm 0.42$ \\
\hline $\mathrm{Cr}+\mathrm{MCAO}$ & $41.4 \pm 1.83^{\# \# \#}$ & $27.9 \pm 3.11^{\#}$ & $17.1 \pm 0.82^{\# \#}$ & $63.8 \pm 1.05^{\# \# \#}$ \\
\hline ATRA + MCAO & $20 \pm 1.97$ & $15.6 \pm 1.83$ & $7.10 \pm 1.04$ & $112.3 \pm 0.52$ \\
\hline $\mathrm{Cr}+\mathrm{ATRA}+\mathrm{MCAO}$ & $17.8 \pm 2.24$ & $13.7 \pm 2.06$ & $9.1 \pm 1.15$ & $96.3 \pm 0.73$ \\
\hline
\end{tabular}

Carveol significantly normalized the cortex antioxidant enzyme levels. Symbols *** shows significant difference $p<0.001$ while symbol \#\# represents $p<0.01$ values for significant difference. All data were presented as means \pm SEM and analyzed by one-way ANOVA followed by post hoc Bonferroni multiple comparison test. Symbols * show a significant difference of MCAO relative to sham and \# shows a significant difference of MCAO ( $n=5 / g r o u p)$. After euthanasia, samples were collected and preserved at $-80^{\circ} \mathrm{C}$. Abbreviations: GST, glutathione S-transferase; GSH, glutathione; TBARS, thiobarbituric acid reactive substances; LPO, lipid peroxidation.

\#\#\#Indicates $p<0.001$ and it shows significant difference relative to MCAO. 
intensity and analyze p-JNK, COX-2, HO-1, Nrf2, p-NFkB, Bcl2, and caspase 3 positive cells at the same threshold intensity for all groups. The intensity is expressed as the relative integrated density of the samples relative to the control.

\section{Immunofluorescence Analysis}

Slides were treated with proteinase $\mathrm{K}$ (antigen retrieval step), washed with $0.1 \mathrm{M}$ PBS, and incubated with 5\% normal serum according to the source of the secondary antibody used. The slides were incubated with primary antibodies against Nrf2 (dilution: 1:100, Santa Cruz Biotechnology), overnight at $4^{\circ} \mathrm{C}$. The next morning, after being washed with PBS, the slides were incubated with fluorescently labeled secondary antibodies (dilution: 1:50, Santa Cruz Biotechnology) for signal amplification in a dark chamber, then coverslipped in Ultra Cruz mounting medium (Santa Cruz Biotechnology, Inc.). Immunofluorescence images (five images per slide) were captured using fluorescent scanning microscopes (fluorescence microscope), and the same region of the cortex/total area for all groups was quantitated as above.

\section{Oxidative Enzymes Analysis}

Oxidative stress markers such as GSH and glutathione $S$-transferase (GST) levels were determined to assess the degree of oxidative damage and the relative effect of the testing drug. GSH was determined using a previously reported method with slight modifications (Imran et al., 2020). Test drug, phosphate buffer solution and freshly prepared DTNB solution were added together and change in the color was measured at $412 \mathrm{~nm}$ using a spectrophotometer. Phosphate buffer was used as blank whereas the DTNB solution was used as control. Increased absorbance of the mixture revealed the presence of GSH in the test drug. Real absorbance was calculated by subtracting the absorbance of control from that of the sample. Final GSH values were expressed in $\mu$ moles GSH/mg of sample. Likely, for the determination of GST, the previously reported protocol was followed. GSH and CDNB were mixed with the test drug and the optical density of the reaction mixture was recorded at $340 \mathrm{~nm}$ against phosphate buffer blank in a spectrophotometer. Assay mixture without drug served as control. Increased absorbance indicated the presence of GST and the antioxidant properties

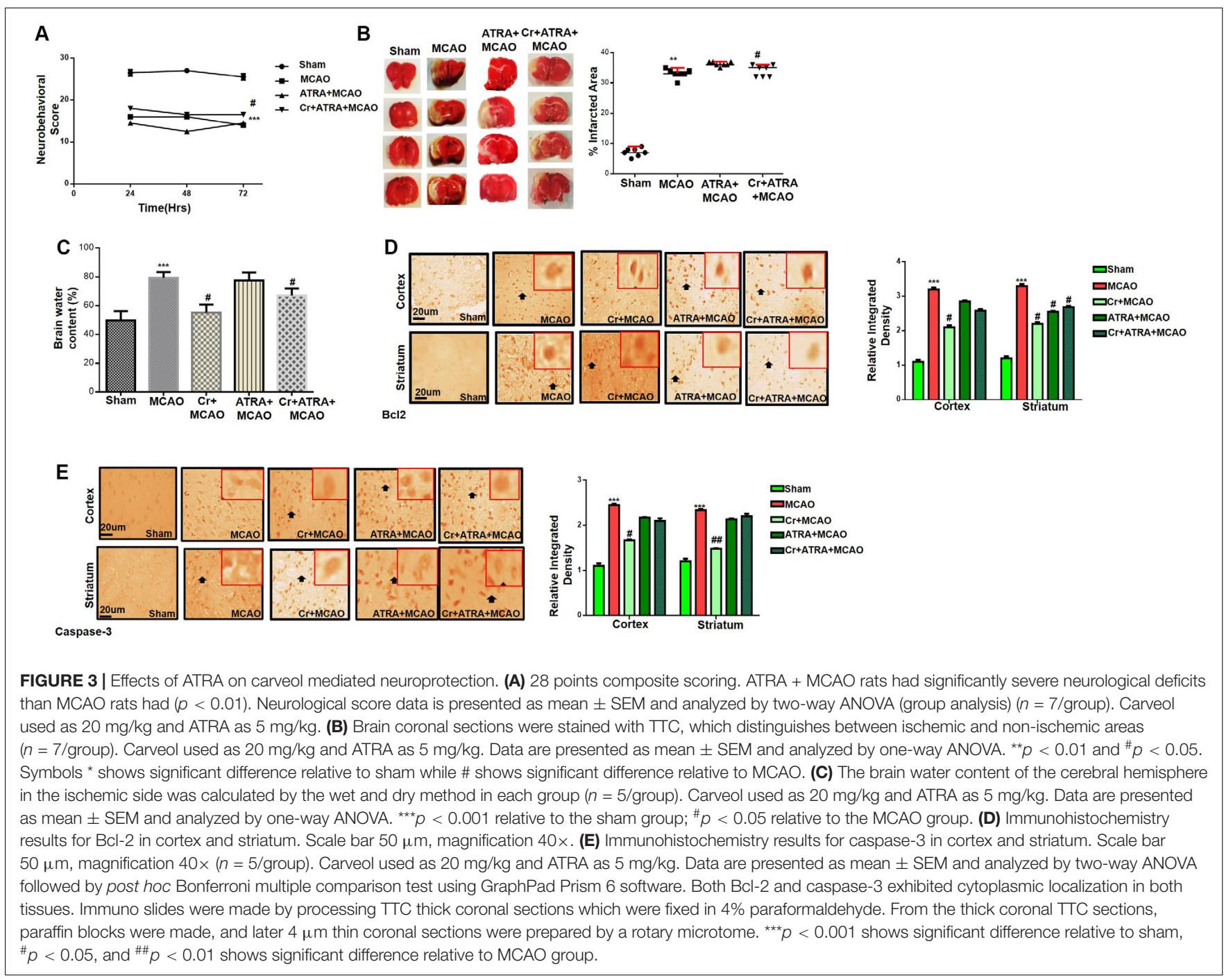


of the sample. GST activity was calculated using the extinction coefficient of the product formed and expressed as $\mu$ moles of $\mathrm{CDNB}$ conjugated $/ \mathrm{min} / \mathrm{mg}$ of protein.

\section{LPO Assay}

Lipid peroxidation (LPO) assay was carried out by measuring thiobarbituric acid reactive substances (TBARS) using fluorimetry as described previously with slight modifications (Ullah et al., 2020). Rats forebrain was homogenized in $10 \mathrm{~mL}$ of $20 \mathrm{mM}$ Tris- $\mathrm{HCl}(\mathrm{pH} 7.4)$ at $4^{\circ} \mathrm{C}$ in a polytron homogenizer. Homogenate was then centrifuged at $1,000 \mathrm{~g}$ for $10 \mathrm{~min}$ at $4^{\circ} \mathrm{C}$ and supernatant was isolated. Freshly prepared ferric ammonium sulfate was then added to this supernatant $(40 \mu \mathrm{L})$ and incubated at $37^{\circ} \mathrm{C}$ for $30 \mathrm{~min}$. Finally, $75 \mu \mathrm{L}$ of thiobarbituric acid (TBA) was added and absorbance was measured immediately at $532 \mathrm{~nm}$ using a microplate reader and expressed as Tbars- $\mathrm{nM} / \mathrm{min} / \mathrm{mg}$ protein.

\section{Enzyme-Linked Immunosorbent Assay (ELISA)}

The Nrf2, HO-1, p-NFкB, TNF- $\alpha$ expression was measured using Rat Nrf2, HO-1, p-NFкB, TNF- $\alpha$ enzyme-linked immunosorbent assay (ELISA) kit according to the manufacturer's instructions (for detail chemicals and reagents). The tissues (approximately $50 \mathrm{mg}$ ) were homogenized at 15,000 RPM using Silent Crusher M (Heidolph) and the supernatant was collected after centrifugation (at $4,000 \times g$ for $10 \mathrm{~min}$ ). The total protein concentration in each group was determined by the BCA method (Elabscience) and the equivalent quantity of protein was then loaded to determine the concentration of Nrf2, HO-1, p-NFאB, and TNF- $\alpha$ by using ELISA microplate reader (BioTek ELx808). and finally, the concentrations $(\mathrm{pg} / \mathrm{ml})$ were then normalized to total protein content (pg/mg total protein).

\section{Docking Studies}

Genetic Optimization of Ligand Docking (GOLD v5.2.2) package was used for docking. The 3D structure of Keap1 (PDB ID: 3CGJ) in complex with RA839 inhibitor was taken from protein data bank (Winkel et al., 2015). All water molecules, heteroatoms, and other unwanted molecules were removed from the Keap1 structure before docking simulation. Hydrogen atoms were added and the docking site of Keapl was traced from the bound inhibitor (RA839). The 2D structures of carveol were taken from PubChem and prepared for docking in Discovery Studio $v 4.5$ (DS). During the docking protocol, a total of 100 conformational poses of carveol were generated using the Genetic
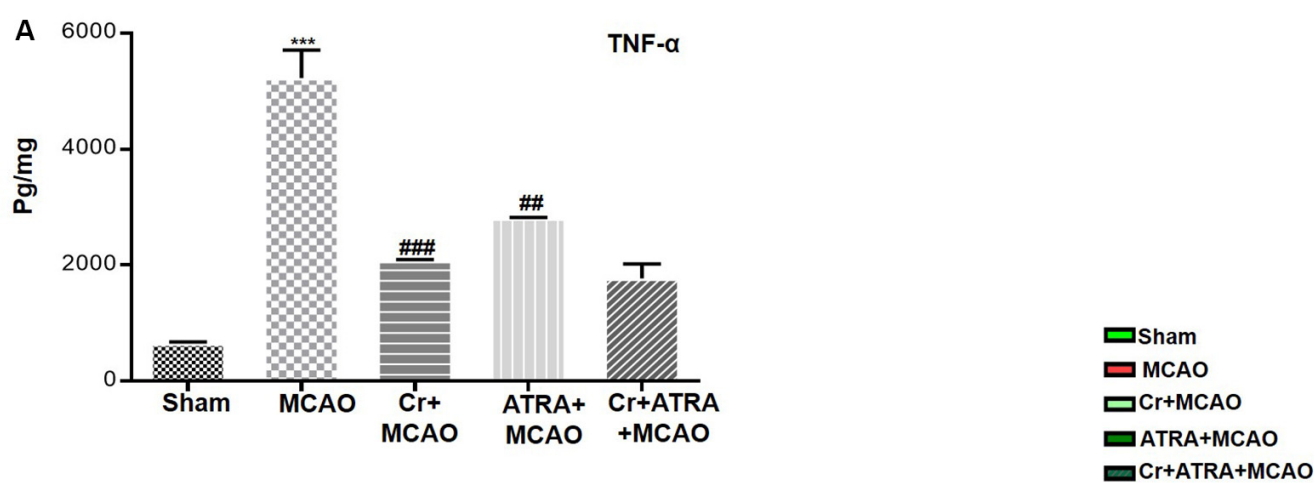

B

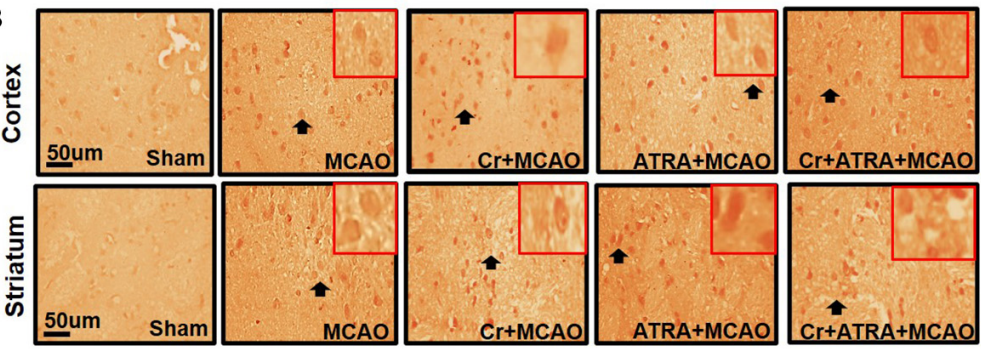
p-JNK

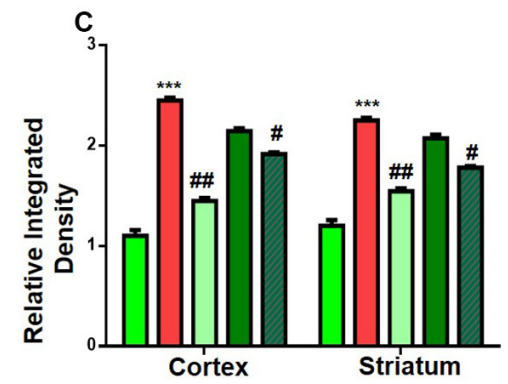

FIGURE 4 | Carveol $(20 \mathrm{mg} / \mathrm{kg})$ ameliorated oxidative stress and neuroinflammation. (A) TNF- $\alpha$ expression was quantified by ELISA in the cortex. The data were expressed as the mean \pm SEM and analyzed by one-way ANOVA. ${ }^{* \star} p<0.001$ relative to the sham while ${ }^{\# \#} p<0.01$ relative to the MCAO with $n=5 /$ group. After euthanasia, samples were collected and preserved at $-80^{\circ} \mathrm{C}$ and later processed for protein analysis. (B) Immunohistochemistry results for pJNK in cortex and striatum tissues of the brain. Scale bar $50 \mu \mathrm{m}$, magnification $40 \times(n=5 /$ group). p-JNK exhibited cytoplasmic localization in both tissues. Histograms show a comparatively higher expression of $\mathrm{p}$-JNK in various segments of the MCAO and ATRA groups. The data were expressed as the mean \pm SEM and analyzed by two-way ANOVA followed by post hoc Bonferroni multiple comparison test using GraphPad Prism 6 software. ${ }^{* \star} p<0.001$ shows significant difference relative to sham, while ${ }^{\#} p<0.05,{ }^{\# \#} p<0.01$ and ${ }^{\# \# \#} p<0.001$ shows significant difference relative to MCAO group. Immuno slides were made by processing $\Pi \mathrm{ITC}$ thick coronal sections which were fixed in $4 \%$ paraformaldehyde. From the thick coronal TTC sections, paraffin blocks were made, and later $4 \mu \mathrm{m}$ thin coronal sections were prepared by a rotary microtome. 
Algorithm (GA) module of the GOLD package. Furthermore, the piecewise linear potential (ChemPLP) was used as the scoring function and the results were evaluated. The promising inhibitory pose of carveol was determined by the highest ChemPLP and polar and non-polar interactions with the crucial residues of Keap1. The docking results were analyzed using DS and GOLD software.

\section{Statistical Analysis}

The data are presented as means \pm SEM. Data were analyzed by one-way or two-way analysis of variance (ANOVA) followed by a post hoc Bonferroni multiple comparison test using GraphPad Prism 6 software. Moreover, behavior studies were analyzed by grouped two-way analysis. Immunohistochemical data and TTC data were analyzed using ImageJ software ${ }^{1}$ (Image J 1.30). In all analyses, differences were considered significant at $p<0.05$. The symbol $*$ indicates a significant difference relative to sham, \# shows a significant difference relative to MCAO.

${ }^{1}$ https://imagej.nih.gov/ij/

\section{RESULTS}

\section{Effect of Post-treatment Dosage Regimen of Carveol on Brain Infarction and Neuronal Cell Loss}

The potential effects of carveol on the chronic pathological and neurological changes after MCAO were first examined. The neurological score was determined daily for $72 \mathrm{~h}$. As shown in Figure 2A, MCAO animals exhibited severe neurological deficits as demonstrated by deficits of cortical and striatal controlling functions, such as sensory and motor coordination (Figure 2A, $p<0.001$ ), which was significantly reversed by daily carveol treatment in a dose-dependent manner (Figure 2A). Specifically, $20 \mathrm{mg} / \mathrm{kg}$ carveol showed improved protective efficacy to reverse the neurological deficits after $72 \mathrm{~h}$ of MCAO (Figure 2A, $p<0.01)$ as compared to $10 \mathrm{mg} / \mathrm{kg}$ of carveol. TTC staining was performed to further evaluate the pathological changes and cellular viability. MCAO produced a significantly larger infarction area as compared to sham-operated group $(p<0.001$, Figure 2B), while carveol treatment significantly attenuated the infarction in a dose-dependent manner $(p<0.05, p<0.01$, Figure 2B), with the corrected infarct area of $33.14 \pm 0.59 \%$

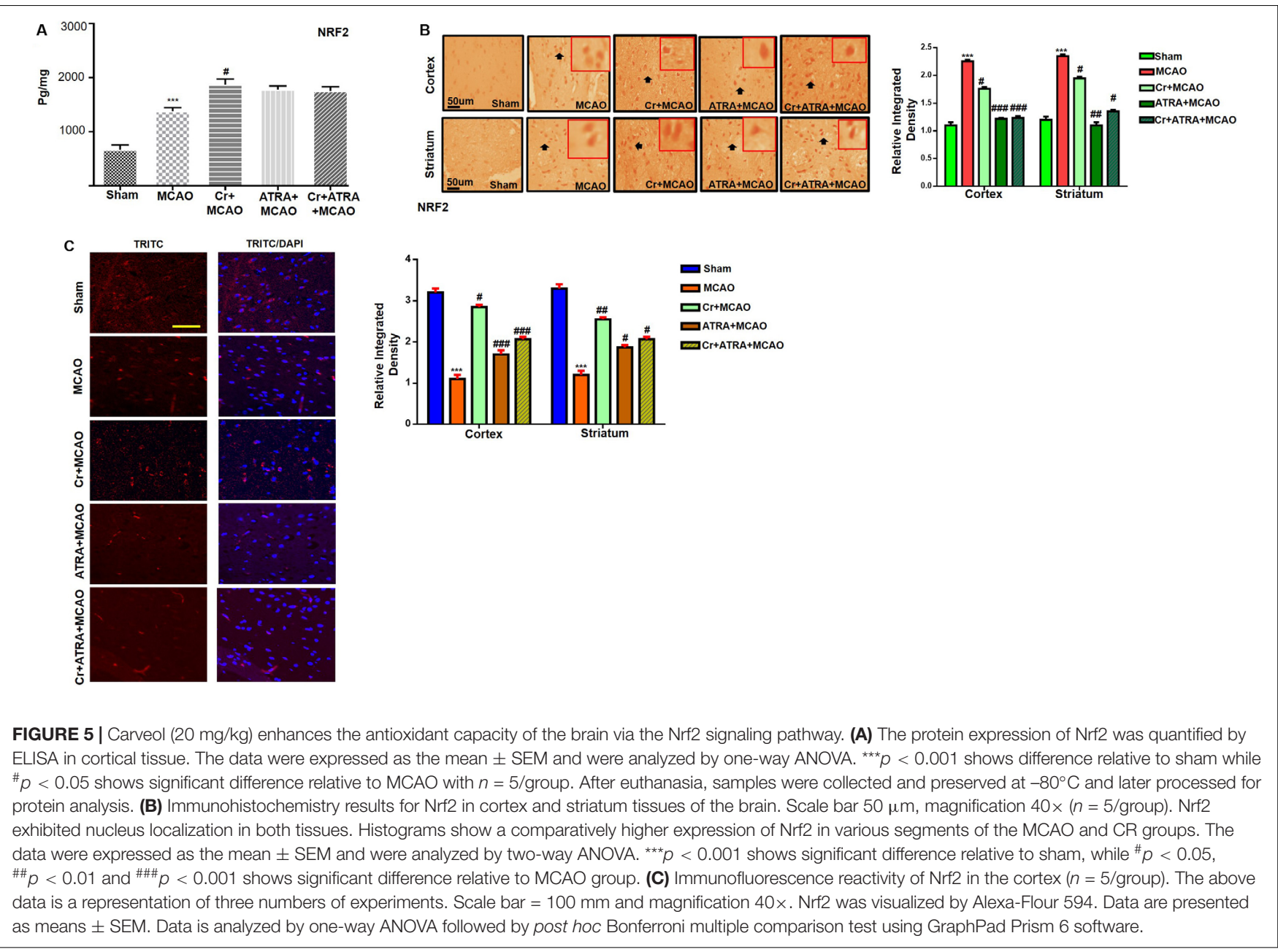


in $\mathrm{MCAO}, 26.5 \pm 0.8 \%$ at $10 \mathrm{mg} / \mathrm{kg}$, and $19.2 \pm 0.86 \%$ at $20 \mathrm{mg} / \mathrm{kg}$ in $\mathrm{Cr}+\mathrm{MCAO}$ groups (Figure 2B), respectively. H\&E staining was used to reveal the extent of neuronal injury in cortex and striatum $72 \mathrm{~h}$ after occlusion and to further examine the neuroprotective effects of carveol. The peri-infarct frontal cortex and striatum were visualized for morphological analysis Figure 2C. As shown in (Figures 2C,D), considerable variations were observed in color staining, neuronal shape, and neuronal integrity after MCAO. Further evaluation of color staining and vacuole formation indicated robust detrimental changes in the ipsilateral cortex and striatum (Figure 2D, $p<0.001$ ), whereas carveol treatment attenuated the damage and increased number of intact neuronal cells could be seen (Figure 2D, $p<0.001$ ).

\section{Effects of ATRA on Carveol Mediated Neuroprotection}

Several studies demonstrated the inhibitory effect of ATRA on the Nrf2 signaling pathway and different doses of ATRA was employed to inhibit Nrf2 in several experimental models (Wang X. J. et al., 2007). We then investigated whether Nrf2 could be involved in the underlying neuroprotective effects of carveol. Based on our preliminary results (data not shown), $5 \mathrm{mg}$ ATRA was found to inhibit Nrf2 in our study, and this inhibition by ATRA further exacerbates the MCAO induced damage as shown (Table 1). These results were further validated via neurobehavioral defects (Figure 3A), whereas composite score was reduced to $(16.8 \pm 0.73)$ in $\mathrm{Cr}+$ ATRA + MCAO group like that of MCAO group $(15.7 \pm 0.99)$ (Figure 3A) and with the corrected infarct area to be $(33 \pm 0.59 \%)$ and $(36 \pm 0.50 \%)$, respectively in MCAO and $\mathrm{Cr}+\mathrm{ATRA}+\mathrm{MCAO}$ group (Figure 3B). Meanwhile, the brain water content in the MCAO group was considerably higher relative to the sham group ( $p<0.001$, Figure 3C) whereas the edema in the carveol treated group $(20 \mathrm{mg} / \mathrm{kg})$ was reduced significantly $(p<0.05$, Figure 3C). These data suggest that carveol treatment can mitigate brain edema after t-MCAO and ATRA abrogated carveol effects, supporting the necessary role of Nrf2. To further examine whether t-MCAO induced neuronal apoptosis is accounted for this cell death at the peri-infarct region, we investigated the expression level of caspase- 3 and $\mathrm{Bcl} 2$ in the ischemic frontal cortex and striatum (Figures 3D,E). Our results demonstrated higher caspase- 3 along with a reduced expression of $\mathrm{Bcl} 2$ in the ipsilateral cortex and striatal tissue following $72 \mathrm{~h}$ after MCAO relative to the sham-operated group (Figures 3D,E). Notably, carveol treatment significantly attenuated the expression of caspase-3 $(p<0.05)$ suggesting that carveol could ameliorate the $\mathrm{MCAO}$ induced neuronal apoptosis.

\section{Carveol Ameliorated Oxidative Stress and Neuroinflammation}

Oxidative stress and inflammation are two interrelated pathophysiological processes involved in ischemic stroke that are early initiated and exhibited as a prolonged detrimental effect until the recovery stage. TBARS is a commonly used method to measure lipid peroxidation end product malondialdehyde, a reactive aldehyde produced by lipid peroxidation of
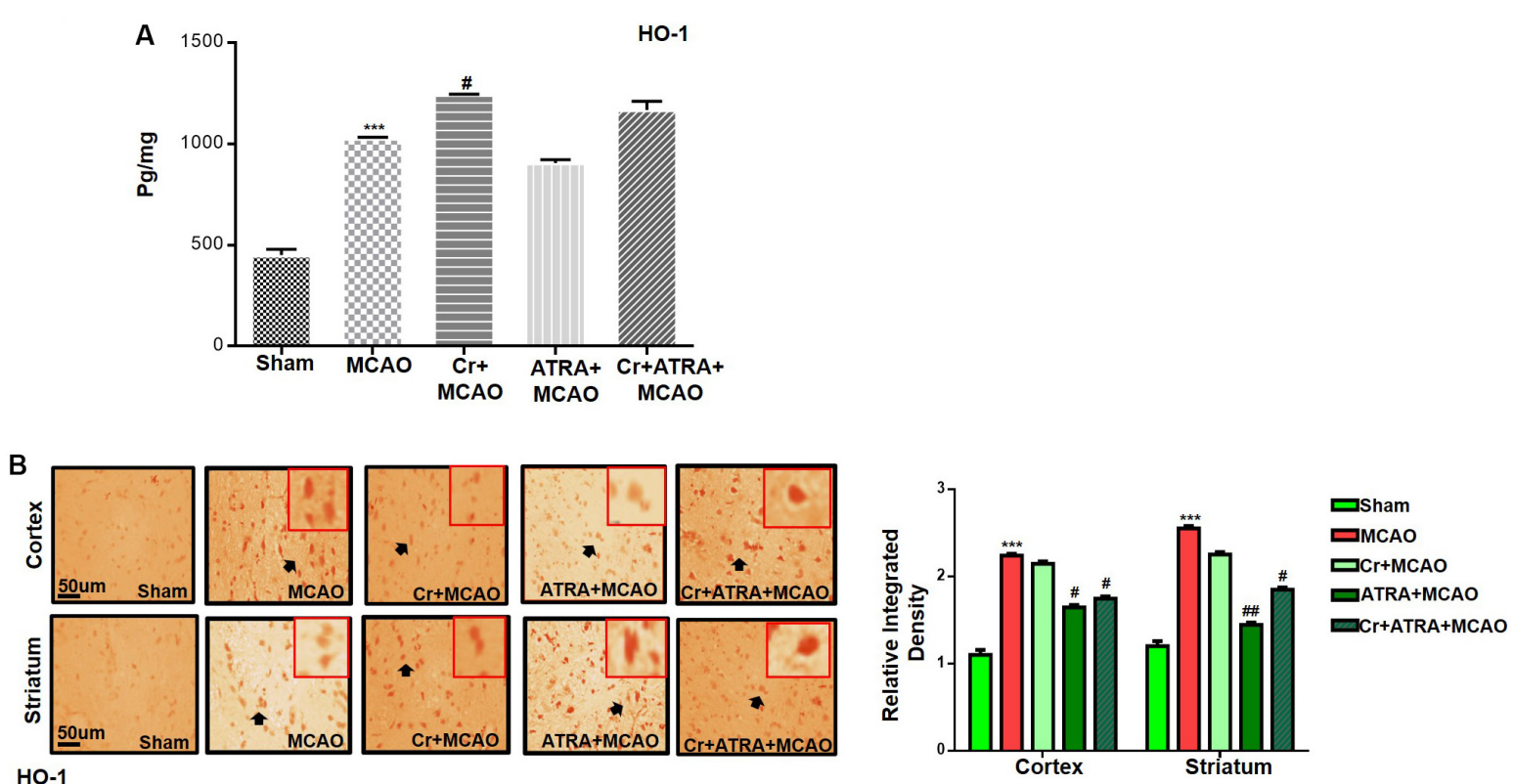

FIGURE 6 | Carveol (20 mg/kg) enhances the antioxidant capacity of the brain via the Nrf2 signaling pathway. (A) ELISA analysis of HO-1 in the cortex. The data were expressed as the mean \pm SEM and analyzed by one-way ANOVA followed by post hoc Bonferroni multiple comparison test using GraphPad Prism 6 software. ${ }^{\star \star \star} p<0.001$ shows difference relative to sham while ${ }^{\#} p<0.05$ shows significant difference relative to MCAO. (B) Immunohistochemistry results for HO- 1 in cortex and striatum tissues of the brain. Scale bar $50 \mu \mathrm{m}$, magnification $40 \times(n=5 /$ group). HO-1 exhibited nucleus localization in both tissues. Histograms show a comparatively higher expression of Nrf2 in various segments of the MCAO and CR groups. ${ }^{\star \star \star} p<0.001$ shows significant difference relative to sham, while \# $p<0.05$ and ${ }^{\# \#} p<0.01$ shows significant difference relative to MCAO group. 
polyunsaturated fatty acids. We then performed the TBARS test and the results showed a drastic increase of peroxides in the MCAO group, an effect that could be rescued by carveol treatment (Table 1). The LPO content in the cortical homogenate was increased to $(119.8 \pm 0.73)$ in the MCAO group as compared to $(40.5 \pm 1.05)$ in the sham-operated group $(p<0.001$, Table 1). A total of $20 \mathrm{mg} / \mathrm{kg}$ carveol significantly $(p<0.05$, Table 1) attenuated this increase $(63.14 \pm 1.05)$ to a level comparable to that of the shamoperated group. We then examined the effects of carveol on inflammatory-related factors. As shown in Figure 4, a higher level of TNF- $\alpha$ could be observed after MCAO, which could be attenuated by carveol treatment $(p<0.001$, Figure 4A) by ELISA analysis. Similarly, JNK, one of the major signaling of the mitogen-activated protein kinase (MAPK) pathway which functions in the control of apoptosis, inflammation, cytokine production, and metabolism, showed a reduced expression after carveol treatment, demonstrating the antioxidative and anti-inflammatory effects of carveol $(p<0.01$, Figure 4B).

\section{Carveol Enhances the Antioxidant Capacity of the Brain via the Nrf2 Signaling Pathway}

Nuclear factor erythroid 2-related factor 2 (Nrf2) is an endogenous antioxidant enzyme which executes vital protective functions (Reisman et al., 2009). Translocation of Nrf2 to nucleus imitates transcription of several downstream antioxidant proteins like heme oxygenase-1 (HO-1), superoxide dismutase (SOD), and GSH to annihilate ROS and protect the cell from inflammation and apoptosis (Ning et al., 2018). Nrf2 also suppressed the expression of proinflammatory factors like $\mathrm{p}-\mathrm{NF} \kappa \mathrm{B}$ and COX2. Thus, to examine the possible effect of carveol on Nrf2 related antioxidant signaling pathway and proinflammatory factors, we measured their expression levels. As

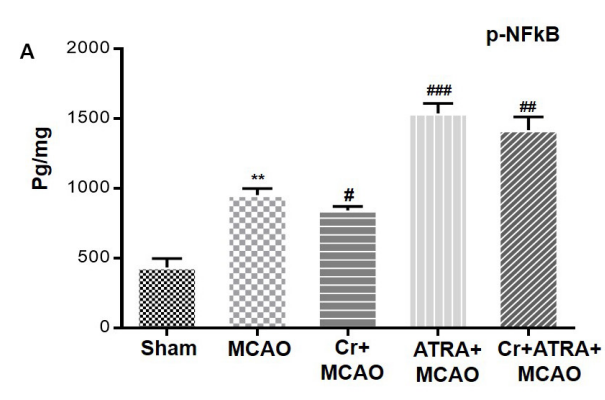

B
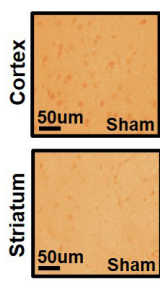

p-NFkB

C
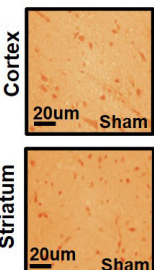

cox-2
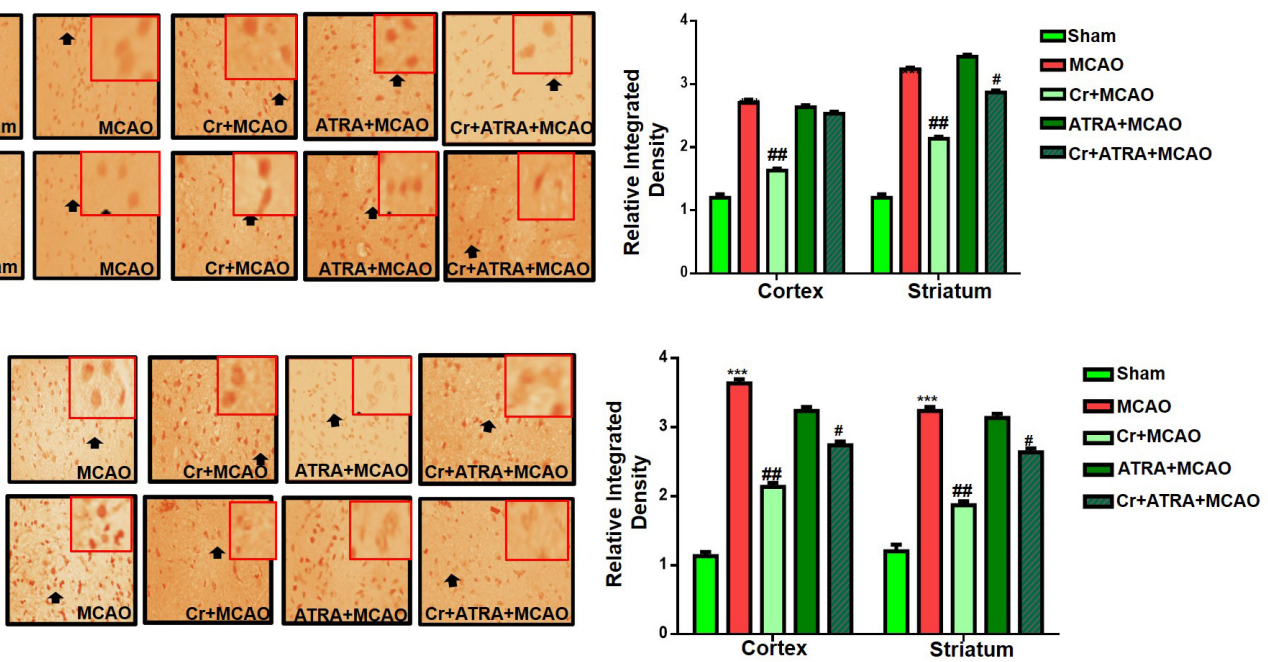

FIGURE 7 | Effect of carveol (20 mg/kg) on outcomes of MCAO induced inflammatory mediators. (A) The protein expression of p-NFKB was quantified by ELISA in the brain cortex. The data were expressed as the mean \pm SEM and were analyzed by one-way ANOVA with $n=5 /$ group. ${ }^{* \star} p<0.001$ shows difference relative to sham while ${ }^{\#} p<0.05,{ }^{\# \#} p<0.01$, and ${ }^{\# \# \#} p<0.001$ shows significant difference relative to MCAO. (B) Immunohistochemistry results for $p-\mathrm{NF} \mathrm{KB}$ in cortex and striatum tissues of the brain. Scale bar $50 \mu \mathrm{m}$, magnification $40 \times$. (C) Immunohistochemistry results for COX-2 in the cortex and striatum tissues of the brain. Scale bar $50 \mu \mathrm{m}$, magnification $40 \times(n=5 /$ group). p-NFKB exhibited nucleus localization while COX-2 exhibited cytoplasmic localization in both tissues of the brain. Histograms show comparatively higher expression of $\mathrm{p}-\mathrm{NF} \kappa \mathrm{B}$ and $\mathrm{COX}-2$ in various segments of the MCAO and ATRA groups. ${ }^{\star \star *} p<0.001$ shows significant difference relative to sham, while ${ }^{\#} p<0.05$ and ${ }^{\# \#} p<0.01$ shows significant difference relative to MCAO group. Data are presented as means \pm SEM. Data is analyzed by two-way ANOVA followed by post hoc Bonferroni multiple comparison test using GraphPad Prism 6 software. Immuno slides were made by processing TTC thick coronal sections which were fixed in $4 \%$ paraformaldehyde. From the thick coronal TTC sections, paraffin blocks were made and later $4 \mu \mathrm{m}$ thin coronal sections were prepared by a rotary microtome. 
shown by ELISA results in Figure 5A, Nrf2 expression was increased in the MCAO group, while carveol treatment further increases their expression $(p<0.05)$. To further validate our ELISA results, we performed immunohistochemical analysis (Figure 5B) and immunofluorescence analysis (Figure 5C). Likely, HO-1 (Figure 6) GST and GSH showed a similar pattern of changes. The changes in antioxidative enzyme levels of different groups were also summarized in Table $\mathbf{1}$. MCAO induced an elevation in ROS generation, associated with depletion of GSH level (13.65 \pm 0.48$)$, GST activity (10.75 \pm 2.05), and catalase (8.22 \pm 0.96) in the brain cortical tissue $(p<0.001)$. Treatment with carveol attenuated downregulation of GSH (41.4 \pm 1.83$)$, GST (27.9 \pm 3.1$)$, and catalase $(17.1 \pm 0.82)$, which is comparable to the shamoperated group.

\section{Effect of Carveol on Outcomes of MCAO Induced Inflammatory Mediators}

Pro-inflammatory factors TNF- $\alpha$ and IL- $1 \beta$ binding to respective receptors triggered sequential activation of downstream molecules such as ASK1, SEK1, and JNK. Collectively, this leads to proteasomal dependent IкB dissociation, and nuclear translocation of $\mathrm{NF}_{K} \mathrm{~B}$ to induce inflammatory transcription types of machinery like NOS2 and COX2 (Davies et al., 2016). These proteins were hyper expressed in ELISA findings in the ischemic brain $(p<0.01$, Figure 7A), and the carveol post-treatment dosage regimen significantly alleviated the expression $(p<0.05)$. These results were further validated by immunostaining findings for $\mathrm{p}-\mathrm{NF}-\kappa \mathrm{B}$, and COX2 (Figures 7B,C).

\section{Bioinformatics Analysis}

The above experimental results suggested that carveol activates Nrf2 signaling, which is localized to the cytoplasm as an inactive dimer with keap1. Therefore, we then performed docking studies of carveol and Keap1 to examine the possibility that carveol may bind Keap1 to disrupt its interaction with Nrf2 and facilitate its migration to the nucleus. The docking results demonstrated that carveol could bind Keap1 and orient in the Nrf2 binding site. Since the Nrf2 binding site of Keap1 is shallow and subdivided into the acidic region, the planner acceptor region and sulfamide pocket (Figure 8) (Winkel et al., 2015). Therefore, we asked the suitable sub-site and binding orientation of carveol in Keap1. Our results demonstrated that carveol oriented in two different stoichiometric conformations in the Nrf2-binding site of Keap1 (Figures 9B,C). Carveol occupied the acidic region (Figure 8B) and sulfamide pocket (Figure 8C) of the Nrf2binding site of Keap1 and obtained a docking score of 33.88 and 35.18 , respectively (Table 2 ).

Furthermore, the molecular interaction analysis suggested that carveol established hydrogen bond interaction with Arg483 in the acidic region of Keap1 (Figures 9B,E). In the acidic region of Keap1, carveol also formed another hydrogen bond with Ser508 (Figures 9B,E). Moreover, the molecular interaction analysis of carveol in the sulfamide region established a hydrogen bond with Ser 602 of Keap1 (Figures 9C,F). Collectively, we speculate that carveol may orient in the acidic as well as sulfamide sub-pockets of Keap1 and forms hydrogen bonds with Arg483 and Ser602, respectively. Apart from the hydrogen bond formed with key residues of Keap1, carveol could also establish nonpolar interactions with Nrf2 binding residues of Keap1 (Figure 9,

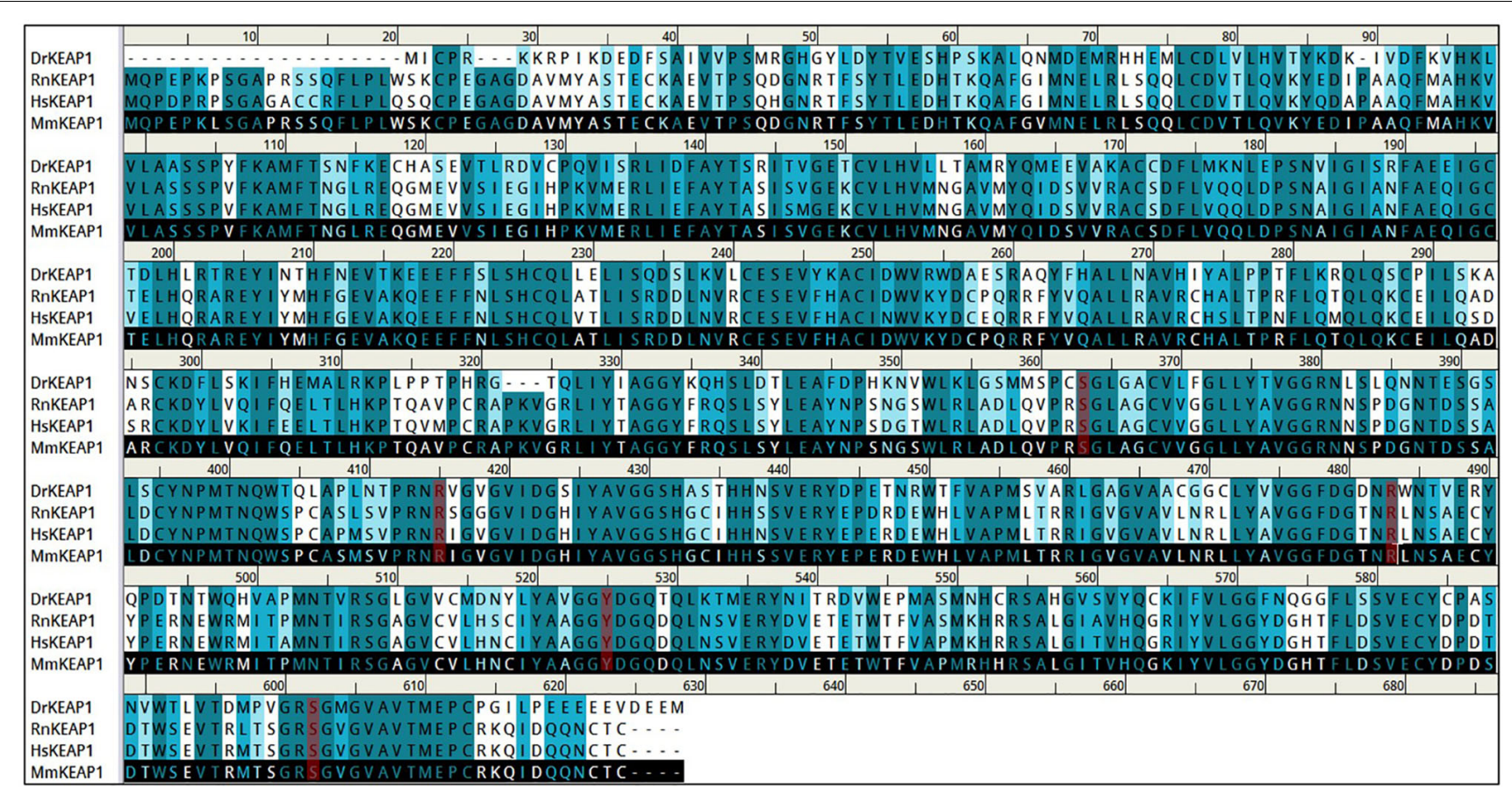

FIGURE 8 | Arg483, Ser363, Arg525, and Ser602 are conserved residues in the Nrf2-binding site of Keap1. 

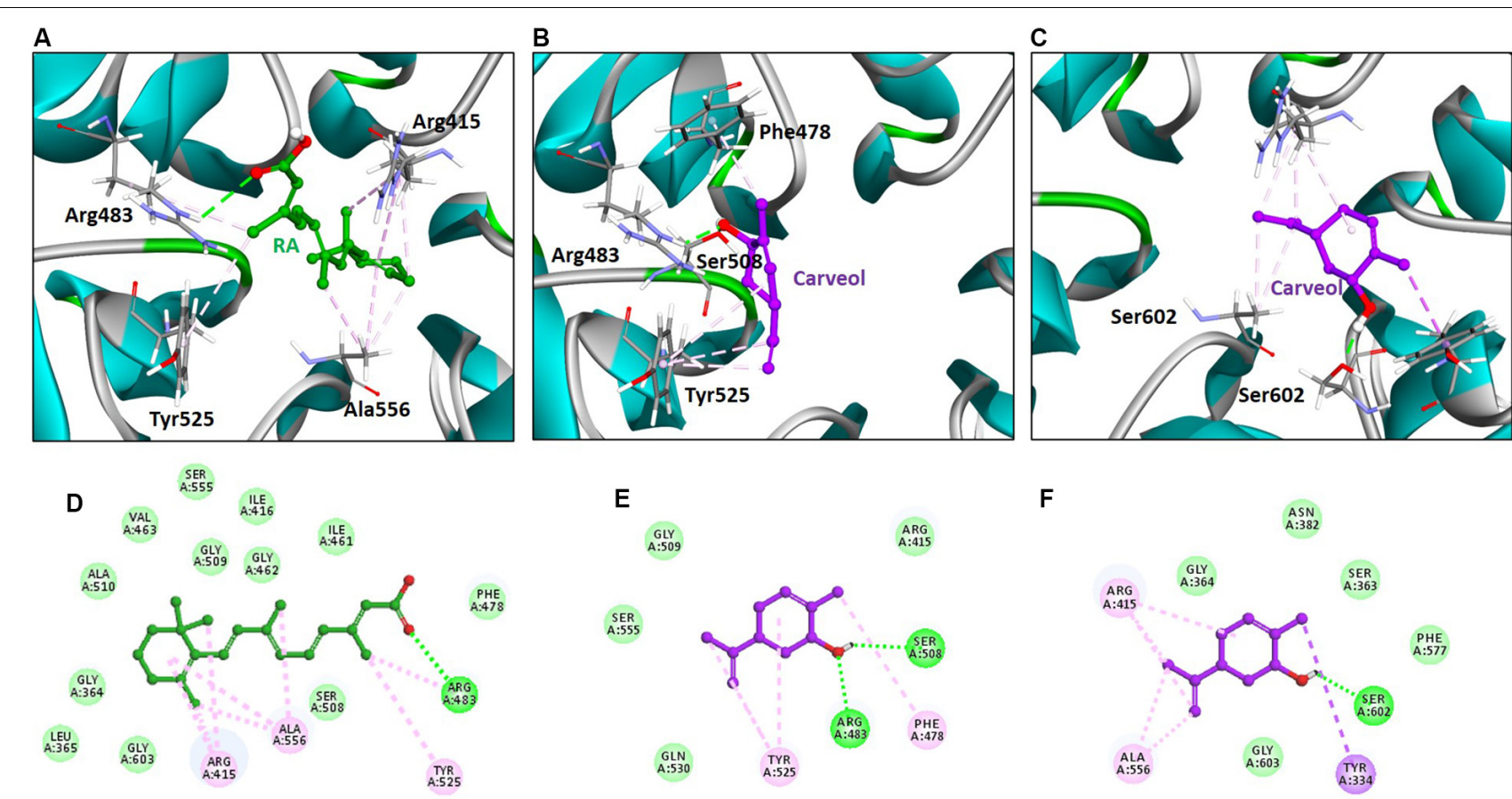

FIGURE 9 | Retinoic acid and carveol occupy the Nrf2-binding site of Keap1. The upper panel (A-C) shows the binding orientation of retinoic acid and carveol in the Nrf2-binding site of Keap1. (A) Retinoic acid established hydrogen bond with Arg483 of Keap1. (B) One carveol molecule formed a hydrogen bond with Arg83 and Ser508 in the acidic region of Keap1. (C) Another carveol molecule established a hydrogen bond with Ser602 in the sulfamide region of Keap1. Retinoic acid and carveol are represented as ball and stick models and colored as green and magenta, respectively. Keap1 residues participated in either polar or non-polar interactions are depicted as stick representation. Hydrogen bonds are represented as green color dashed lines. Other hydrophobic interactions are portrayed as light magenta-colored dash lines. Lower panel (D-F) displayed the 2D (two dimensional) interaction pattern of Retinoic acid and carveol with Keap1. (D) Retinoic acid, (E) carveol in the acidic region of Keap1, and (F) carveol in the sulfamide region of Keap1 formed hydrogen bonds with Keap1. Retinoic acid and carveol are presented as ball and stick models and colored as green and magenta, respectively. Hydrogen bonds forming residues are represented as green color circles and labeled. Hydrogen bonds are displayed as green-colored dash lines. Other non-polar interacting residues are depicted as light green colored (residues forming Van der Waals interactions) and light magenta-colored (hydrophobic interactions) and labeled.

Table 2). For instance, Tyr525 formed hydrophobic interaction with carveol which has also been reported for other small molecules inhibitors (Marcotte et al., 2013). In the sulfamide region, carveol formed $\pi-\sigma$ interaction with Tyr334 and alkyl interactions with Arg415 and Ala556 (Figures 9C,F). Apart from polar interaction with Arg415, carveol established several non-polar (hydrophobic and Van der Waals interactions) with the Nrf2 binding site residues of Keap1 (Figure 9, Table 2). Therefore, we demonstrate that carveol occupies the Nrf2 binding site of Keap1 and reduces the binding frequency of Nrf2 and Keap1.

\section{DISCUSSION}

The natural drug substances are significantly investigated for their therapeutic potentials including in various neurodegenerative models both to understand the pathophysiology and to develop better therapeutic options. In the present study, our objective is to investigate for the first time (as per our knowledge and literature survey) the neuroprotective effects of naturally derived substance carveol against MCAO induced oxidative stress and neurodegeneration. Carveol attenuated MCAO induced apoptosis and neurodegeneration both in cortical and striatal tissues after $72 \mathrm{~h}$ of transient ischemic injury. These beneficial and neuroprotective effects are likely mediated by mitigating neuroinflammation, oxidative stress, and enhancing free radical scavenging activity. Further, our results demonstrated that carveol act as a potent antioxidant and modulator of Nrf2, which stimulated the other antioxidative mechanism, including GSH and HO-1, and reducing pro-neuroinflammatory factors and mediators such as p-NF-кB (Figure 10).

Post-ischemic treatment reveals a more valuable and clinically significant approach both for understanding the pathophysiology and to develop better therapeutic options over a longer period. Excessive accumulation of ROS is an established and wellstudied pathological mechanism in experimental ischemic stroke (Kurian et al., 2016), and which is further associated with different cellular signaling, implicated in neuroinflammation and thus further intensifies the pathogenesis of ischemic injury (Aguilera et al., 2018; Solleiro-Villavicencio and Rivas-Arancibia, 2018; Kishimoto et al., 2019). Therefore, oxidative stress is a rational avenue to investigate the anti-oxidative potential of natural drug substances (Li and Yang, 2016; Yang et al., 2019). Our results showed that carveol reduced the elevated oxidative 


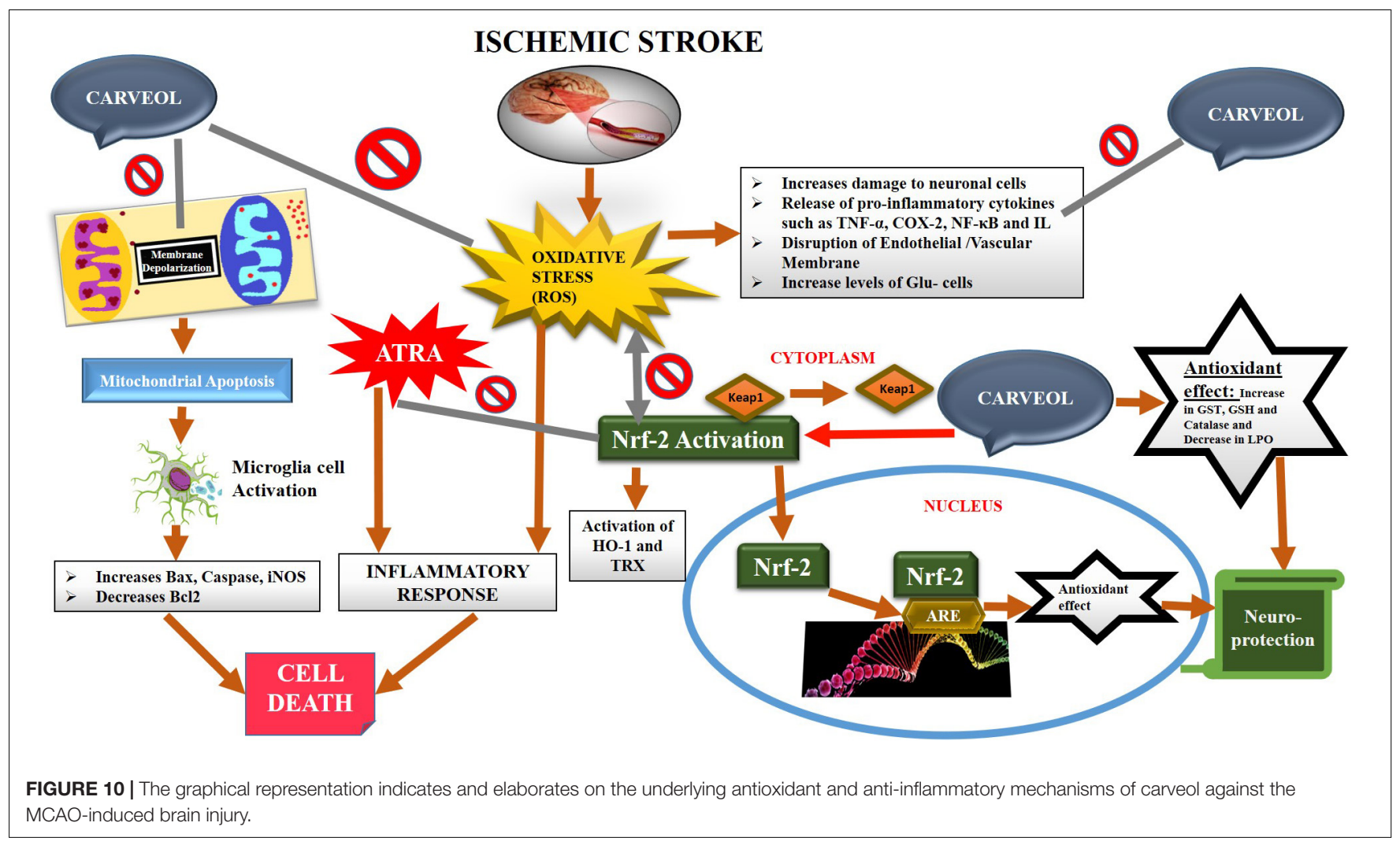

TABLE 2 | Docking scores and hydrophobic interactions of retanoic acid and carveol with Keap-1.

\begin{tabular}{|c|c|c|c|c|c|c|}
\hline \multirow[t]{2}{*}{ Inhibitor } & \multirow[t]{2}{*}{ Docking score } & \multicolumn{4}{|c|}{ Hydrogen bonds (<3.5 A) } & \multirow[t]{2}{*}{ Hydrophobic interactions } \\
\hline & & Amino acid & Amino acid atom & Ligand atom & Distance (Å) & \\
\hline Retanoic Acid & 54.19 & Arg483 & $\mathrm{HE}$ & 02 & 3.0 & $\begin{array}{l}\text { Arg415, Ala556, Tyr525, Ser508, Phe478, lle461, } \\
\text { Gly462, Ile416, Gly509, Ser555, Val463, Ala510, } \\
\text { Gly364, Leu365, Gly603 }\end{array}$ \\
\hline Carveol $A^{*}$ & 33.88 & Arg483 Ser508 & HE OG & $\mathrm{O} 1 \mathrm{H} 25$ & 2.01 .9 & Tyr525, Phe478, Arg415, Gly509, Ser555, Gln530 \\
\hline Carveol $S^{\#}$ & 35.18 & Ser602 & OG & $\mathrm{H} 25$ & 1.7 & $\begin{array}{l}\text { Arg415, Ala556, Tyr334, Gly603, Phe577, Ser363, } \\
\text { Asn382, Gly364 }\end{array}$ \\
\hline
\end{tabular}

${ }^{*}$ Carveol A: Carveol in the acidic region. "Carveol S: Carveol in sulfamide region. Abbreviations: Gly, glycine; Ala, alanine; Ser, serine; Tyr, tyrosine; Ile, iso-leucine; Arg, arginine; Phe, phenylalanine; Val, valine; Leu, leucine; Gln, glutamine.

stress, coherent with the previous study, whereas the carveol has been reported to exhibit antioxidant and anti-hyperlipidemic activities (Johri, 2011). Further, we assessed that this antioxidative activity of carveol may be attributed to Nrf2 activation and its downstream HO-1 targets which subsequently regulate and mobilize other endogenous antioxidant systems, such as SODs, GSH, and sirtuins (Sirt). In our study, antioxidant activity in the ischemic cortex was assessed and increased levels of GST, GSH, and catalase were observed associated with reduced LPO level, illustrating its antioxidant nature in our ischemic model. The detailed molecular mechanisms of carveol effects are still unidentified but could be partially attributed to its free radical scavenging properties. Previous studies reveal that the Nrf2 network plays a central role in the cellular adaption by dealing with a wide range of cytoprotective proteins, counteracting distinct endogenous and exogenous insults, and providing a promising optimal therapeutic target against multiple diseases from cancer to vascular and brain disorders (Dinkova-Kostova et al., 2018; Sivandzade et al., 2019; Panieri and Saso, 2019; Brandes and Gray, 2020). The role of the Nrf2 in ischemic stroke and its underlying neuroprotection has also been evidenced using different ischemic stroke rodent models (Wang X. J. et al., 2007; Aguilera et al., 2018; Solleiro-Villavicencio and Rivas-Arancibia, 2018; Kishimoto et al., 2019).

The oxidative stress associated kinases such as activated p-JNK (MAPK) signaling has a pivotal role in neuroinflammation and apoptotic neurodegeneration (Choudhury et al., 2015; Chen et al., 2018). Numerous studies reported that suppression and inhibition of p-JNK could attenuate neuroinflammation and neurodegeneration not only in ischemic stroke but also in 
other neurodegenerative models (Atochin et al., 2016; Zheng et al., 2020). Interestingly, here we also found that carveol downregulated the activated p-JNK in the ischemic brain compared to the sham control, which described that the carveol neuroprotective effect may be partially associated with the regulation of MAPKs.

A strong relationship between inflammation and oxidative stress is revealed in both neuronal and non-neuronal models (Yeligar et al., 2010; Ali et al., 2018). The release of inflammatory factors (IL-1b and IL-6) are abruptly enhanced at least $48 \mathrm{~h}$ after MCAO (Clausen et al., 2017). Other than these elevated pro-inflammatory cytokines, inducible pro-inflammatory chemokines such as COX-2, NOS-2, and NF- $\mathrm{BB}$ is also involved in the inflammation pathology of MCAO injury (Itoh and Yamamoto, 2005; McNeill et al., 2015; Kobayashi et al., 2016). Our ELISA results suggest that carveol reduced the expression of TNF- $\alpha, \mathrm{COX}-2$, and NF- $\mathrm{B}$, associated with an increase in nuclear translation of $\mathrm{Nrf} 2$, and induces further other antioxidant genes. Collectively, these processes attenuate inflammation by inhibiting the NF-кB pathway and other pro-inflammatory markers, which can persist for at least 3 days after ischemic injury, as revealed by the 1.8- to 3.6-times increased levels of HO-1, NQO1, SOD2, and GPx proteins after MCAO (Liu et al., 2018). Our results that ATRA treatment abolished the neuroprotective effects of carveol further supported the pivotal role of $\mathrm{Nrf} 2$ as a modulator of both oxidative stress and neuroinflammation. In addition, our results are coherent with the previous observation which supported the key role of Nrf2 in the neuroinflammation. The Nrf2 overexpression or pharmacological activation rescued neuroinflammation while inhibition of Nrf2 escalated release of pro-inflammatory markers and neuroinflammation in several ischemic and other neurodegenerative models (Kobayashi et al., 2016; Yang et al., 2017; Liu et al., 2018; Ya et al., 2018; Bahn et al., 2019).

Molecular docking results indicated that carveol may competitively bind Keap 1 . The formation of polar and nonpolar interactions between the small molecule inhibitors and the Nrf2 binding site residues of Keap1 have already investigated (Marcotte et al., 2013; Jnoff et al., 2014; Winkel et al., 2015; Davies et al., 2016; Heightman et al., 2019). Recently, Heightman et al. (2019) identified aryl propionic acid inhibitors that occupy the Nrf2-binding site of Keap1 and form hydrogen bonds with Arg483, Ser602, Ser555, and Gln530. The formation of the hydrogen bond between the small molecule inhibitor and Ser602 has also been reported (Davies et al., 2016) as well as between the small molecule inhibitor (RA839) with Arg483 and Ser508 (Winkel et al., 2015). Although the question of whether carveol could bind to these sites still merit further validation, the small size of carveol enables its conformational freedom and can preferentially orient in the shallow binding site of Keap1. Actually, such stoichiometric conformations of small molecule inhibitors have been reported for cpd15A [2-[(5- ((2,5dimethylbenzene)sulfonyl)-6-oxo-1,6-dihydropyrimidine-2-yl) sulfanyl]-N-(2-(trifluoromethyl)phenyl)acetamide], cpd15B and cpd16 with Keap1 (Marcotte et al., 2013). Not alone for carveol, Davies et al. (2016) has also confirmed that small molecule inhibitors bind the Nrf2-binding site of Keap1 and potentially facilitate the migration of Nrf2 into the nucleus. Previously, Jnoff et al. (2014) identified a small molecule inhibitor (1S,2R)-2-[(1S)-1-[(1,3-dioxo-2,3-dihydro$1 \mathrm{H}$-isoindol-2-yl)methyl]-1,2,3,4-tetrahydroisoquinoline-2carbonyl]cyclohexane-1-carboxylicacid $[(\mathrm{S}, \mathrm{R}, \mathrm{S})-1 \mathrm{a}]$, which could target the Keap1-Nrf2 interaction and occupied the Nrf2-binding site of Keap1.

\section{CONCLUSION}

In summary, our in vivo and molecular docking findings demonstrated that carveol acted as a potent antioxidant agent that triggered activation of principal endogenous antioxidant Nrf2 and ameliorated MCAO induced oxidative stress and neuroinflammation possibly by modulating the p-JNK and other neuroinflammatory mediators. We propose that carveol treatment could be an appropriate therapeutic option to reverse ischemic stroke-induced neuroinflammation and neurodegeneration. Moreover, further research work is required to completely understand the underlying protective mechanism as well as complete pharmacological and pharmacodynamic profile of carveol against stroke-induced neuroinflammation and neurodegeneration.

\section{DATA AVAILABILITY STATEMENT}

All datasets generated for this study are included in the article/Supplementary Material.

\section{ETHICS STATEMENT}

The animal study was reviewed and approved by Institutional Animal Care and Use Committee of Riphah Institute of Pharmaceutical Sciences.

\section{AUTHOR CONTRIBUTIONS}

IM: conceptualization. FS: methodology, investigation, and funding acquisition. TA, NU, and IM: validation. FS and IM: resources. IM, FS, SL, and AK: writing. FS, TA, AA, and RA: review and editing. FS, ZT, and SL: supervision. All authors contributed to the article and approved the submitted version.

\section{FUNDING}

This research was supported partially by the Start-Up Research Grant Program (SRGP), Ministry of Higher Education 
Commission (HEC), Pakistan (21-1615/SRGP/HEC), and grants JCYJ20170810163329510 by Science and Technology Innovation Committee of Shenzhen China and Shenzhen-Hong Kong Institute of Brain Science Shenzhen Fundamental Research Institutions No: 2019SHIBS0004.

\section{REFERENCES}

Aguilera, G., Colín-González, A. L., Rangel-López, E., Chavarría, A., and Santamaria, A. (2018). Redox signaling, neuroinflammation, and neurodegeneration. Antioxid. Redox Signal. 28, 1626-1651. doi: 10.1089/ars.2017.7099

Ali, A., Shah, F., Zeb, A., Malik, I., Alvi, A. M., Alkury, L. T., et al. (2020). NF-кB inhibitors attenuate MCAO induced neurodegeneration and oxidative stressa reprofiling approach. Front. Mol. Neurosci. 13:33. doi: 10.3389/fnmol.2020. 00033

Ali, T., Rehman, S. U., Shah, F. A., and Kim, M. O. (2018). Acute dose of melatonin via Nrf2 dependently prevents acute ethanol-induced neurotoxicity in the developing rodent brain. J. Neuroinflamm. 15:119.

Atochin, D. N., Schepetkin, I. A., Khlebnikov, A. I., Seledtsov, V. I., Swanson, H., Quinn, M. T., et al. (2016). A novel dual NO-donating oxime and c-Jun $\mathrm{N}$-terminal kinase inhibitor protects against cerebral ischemia-reperfusion injury in mice. Neurosci. Lett. 618, 45-49. doi: 10.1016/j.neulet.2016.02.033

Bahn, G., Park, J.-S., Yun, U. J., Lee, Y. J., Choi, Y., Park, J. S., et al. (2019). NRF2/ARE pathway negatively regulates BACE1 expression and ameliorates cognitive deficits in mouse Alzheimer's models. Proc. Natl. Acad. Sci. 116, 12516-12523. doi: 10.1073/pnas. 1819541116

Barone, F. C., and Feuerstein, G. Z. (1999). Inflammatory mediators and stroke: new opportunities for novel therapeutics. J. Cereb. Blood Flow Metab. 19, 819-834. doi: 10.1097/00004647-199908000-00001

Brandes, M. S., and Gray, N. E. (2020). NRF2 as a therapeutic target in neurodegenerative diseases. ASN Neuro 12:1759091419899782. doi: 10.1177/ 1759091419899782

Chen, X., Li, X., Zhang, W., He, J., Xu, B., Lei, B., et al. (2018). Activation of AMPK inhibits inflammatory response during hypoxia and reoxygenation through modulating JNK-mediated NF-кB pathway. Metabolism 83, 256-270. doi: 10.1016/j.metabol.2018.03.004

Choudhury, S., Ghosh, S., Gupta, P., Mukherjee, S., and Chattopadhyay, S. (2015). Inflammation-induced ROS generation causes pancreatic cell death through modulation of Nrf2/NF-кB and SAPK/JNK pathway. Free Rad. Res. 49, 13711383. doi: $10.3109 / 10715762.2015 .1075016$

Chung, I. Y., and Benveniste, E. (1990). Tumor necrosis factor-alpha production by astrocytes. Induction by lipopolysaccharide, IFN-gamma, and IL-1 beta. J. Immunol. 144, 2999-3007.

Clausen, B. H., Lundberg, L., Yli-Karjanmaa, M., Martin, N. A., Svensson, M., Alfsen, M. Z., et al. (2017). Fumarate decreases edema volume and improves functional outcome after experimental stroke. Exper. Neurol. 295, 144-154. doi: 10.1016/j.expneurol.2017.06.011

Crowell, P. L., Kennan, W. S., Haag, J. D., Ahmad, S., Vedejs, E., and Gould, M. N. (1992). Chemoprevention of mammary carcinogenesis by hydroxylated derivatives of d-limonene. Carcinogenesis 13, 1261-1264. doi: 10.1093/carcin/ 13.7.1261

Davies, T. G., Wixted, W. E., Coyle, J. E., Griffiths-Jones, C., Hearn, K., Mcmenamin, R., et al. (2016). Monoacidic inhibitors of the Kelch-like ECHassociated protein 1: nuclear factor erythroid 2-related factor 2 (KEAP1: NRF2) protein-protein interaction with high cell potency identified by fragment-based discovery. J. Med. Chem. 59, 3991-4006.

De Carvalho, C. C., and Da Fonseca, M. M. R. (2006). Carvone: why and how should one bother to produce this terpene. Food Chem. 95, 413-422. doi: 10.1016/j.foodchem.2005.01.003

Dinkova-Kostova, A. T., Kostov, R. V., and Kazantsev, A. G. (2018). The role of Nrf2 signaling in counteracting neurodegenerative diseases. FEBS J. 285, 3576-3590. doi: 10.1111/febs.14379

Dirnagl, U., Iadecola, C., and Moskowitz, M. A. (1999). Pathobiology of ischaemic stroke: an integrated view. Trends Neurosci. 22, 391-397. doi: 10.1016/s01662236(99)01401-0

\section{SUPPLEMENTARY MATERIAL}

The Supplementary Material for this article can be found online at: https://www.frontiersin.org/articles/10.3389/fnins. 2020.00659/full\#supplementary-material

Duan, J., Cui, J., Yang, Z., Guo, C., Cao, J., Xi, M., et al. (2019). Neuroprotective effect of Apelin 13 on ischemic stroke by activating AMPK/GSK-3 $\beta / \mathrm{Nrf} 2$ signaling. J. Neuroinflamm. 16:24.

Gim, S.-A., Lee, S.-R., Shah, F.-A., and Koh, P.-O. (2015). Curcumin attenuates the middle cerebral artery occlusion-induced reduction in $\gamma$-enolase expression in an animal model. Lab. Anim. Res. 31, 198-203.

Gim, S.-A., Sung, J. H., Shah, F.-A., Kim, M. O., and Koh, P. O. (2013). Ferulic acid regulates the AKT/GSK-3 $\beta /$ CRMP-2 signalling pathway in a middle cerebral artery occlusion animal model. Lab. Anim. Res. 29, 63-69.

Heightman, T. D., Callahan, J. F., Chiarparin, E., Coyle, J. E., Griffiths-Jones, C., Lakdawala, A. S., et al. (2019). Structure-activity and structure-conformation relationships of Aryl propionic acid inhibitors of the kelch-like ECH-associated protein 1/nuclear factor erythroid 2-related factor 2 (KEAP1/NRF2) proteinprotein interaction. J. Med. Chem. 62, 4683-4702.

Hennig, P., Garstkiewicz, M., Grossi, S., Di Filippo, M., French, L., and Beer, H.D. (2018). The crosstalk between Nrf2 and inflammasomes. Intern. J. Mol. Sci. 19:562. doi: 10.3390/ijms19020562

Hong, Y., Yan, W., Chen, S., Sun, C.-R., and Zhang, J.-M. (2010). The role of Nrf2 signaling in the regulation of antioxidants and detoxifying enzymes after traumatic brain injury in rats and mice. Acta Pharmacol. Sin. 31, 1421-1430. doi: 10.1038/aps.2010.101

Imran, M., Al Kury, L. T., Nadeem, H., Shah, F. A., Abbas, M., Naz, S., et al. (2020). Benzimidazole containing acetamide derivatives attenuate neuroinflammation and oxidative stress in ethanol-induced neurodegeneration. Biomolecules 10:108. doi: 10.3390/biom 10010108

Iqbal, S., Shah, F. A., Naeem, K., Nadeem, H., Sarwar, S., Ashraf, Z., et al. (2020). Succinamide derivatives ameliorate neuroinflammation and oxidative stress in scopolamine-induced neurodegeneration. Biomolecules 10:443. doi: 10.3390/ biom 10030443

Itoh, K., and Yamamoto, M. (2005). Regulatory role of the COX-2 pathway in the Nrf2-mediated anti-inflammatory response. J. Clin. Biochem. Nutrit. 37, 9-18. doi: $10.3164 /$ jcbn.37.9

Javeed, S., Almas, M., Shahid, M., Sumrin, A., Bashir, H., Bilal, M., et al. (2017). Nrf2: A master regulator of cellular defense mechanism and a novel therapeutic factor. Pak. J. Biotechnol. 14, 121-126.

Jiang, L., Zhang, S., Li, C., Tang, J., Che, F., and Lu, Y. (2017). Roles of the Nrf2/HO1 pathway in the anti-oxidative stress response to ischemia-reperfusion brain injury in rats. Eur. Rev. Med. Pharmacol. Sci. 21, 1532-1540.

Jnoff, E., Albrecht, C., Barker, J. J., Barker, O., Beaumont, E., Bromidge, S., et al. (2014). Binding mode and structure-activity relationships around direct inhibitors of the Nrf2-Keap1 complex. Chemmedchem 9, 699-705. doi: 10. $1002 / \mathrm{cmdc} .201300525$

Johri, R. (2011). Cuminum cyminum and Carum carvi: an update. Pharmac. Rev. 5:63. doi: 10.4103/0973-7847.79101

Kawata, J., Kameda, M., and Miyazawa, M. (2008). Cyclooxygenase-2 inhibitory effects of monoterpenoids with a p-menthane skeleton. Intern. J. Essent. Oil Therap. 2, 145-148.

Kishimoto, M., Suenaga, J., Takase, H., Araki, K., Yao, T., Fujimura, T., et al. (2019). Oxidative stress-responsive apoptosis inducing protein (ORAIP) plays a critical role in cerebral ischemia/reperfusion injury. Sci. Rep. 9, 1-12.

Kobayashi, E. H., Suzuki, T., Funayama, R., Nagashima, T., Hayashi, M., Sekine, H., et al. (2016). Nrf2 suppresses macrophage inflammatory response by blocking proinflammatory cytokine transcription. Nat. Commun. 7, 1-14.

Kurian, G. A., Rajagopal, R., Vedantham, S., and Rajesh, M. (2016). The role of oxidative stress in myocardial ischemia and reperfusion injury and remodeling: revisited. Oxid. Med. Cell. Long. 2016:1656450.

Lee, J.-M., and Johnson, J. A. (2004). An important role of Nrf2-ARE pathway in the cellular defense mechanism. BMB Rep. 37, 139-143. doi: 10.5483/bmbrep. 2004.37.2.139 
Li, W., and Yang, S. (2016). Targeting oxidative stress for the treatment of ischemic stroke: upstream and downstream therapeutic strategies. Brain Circ. 2:153. doi: 10.4103/2394-8108.195279

Liu, L., Locascio, L. M., and Doré, S. (2019). Critical role of Nrf2 in experimental ischemic stroke. Front. Pharmacol. 10:153. doi: 10.3389/fnmol.2020.000153

Liu, L., Vollmer, M. K., Fernandez, V. M., Dweik, Y., Kim, H., and Doré, S. (2018). Korean red Ginseng pretreatment protects against long-term sensorimotor deficits after ischemic stroke likely through Nrf2. Front. Cell. Neurosci. 12:74. doi: 10.3389/fnmol.2020.00074

Mahboubi, M. (2019). Caraway as important medicinal plants in management of diseases. Nat. Prod. Bioprospect. 9, 1-11. doi: 10.1007/s13659-018-0190-x

Marcotte, D., Zeng, W., Hus, J.-C., Mckenzie, A., Hession, C., Jin, P., et al. (2013). Small molecules inhibit the interaction of Nrf2 and the Keap1 Kelch domain through a non-covalent mechanism. Bioorg. Med. Chem. 21, 4011-4019. doi: 10.1016/j.bmc.2013.04.019

McNeill, E., Crabtree, M. J., Sahgal, N., Patel, J., Chuaiphichai, S., Iqbal, A. J., et al. (2015). Regulation of iNOS function and cellular redox state by macrophage Gch1 reveals specific requirements for tetrahydrobiopterin in NRF2 activation. Free Rad. Biol. Med. 79, 206-216. doi: 10.1016/j.freeradbiomed.2014.10.575

Neumann-Haefelin, T., Kastrup, A., Crespigny, A. D., Yenari, M. A., Ringer, T., Sun, G. H., et al. (2000). Serial MRI after transient focal cerebral ischemia in rats. Stroke 31, 1965-1973. doi: 10.1161/01.str.31.8.1965

Ning, C., Gao, X., Wang, C., Kong, Y., Liu, Z., Sun, H., et al. (2018). Ginsenoside $\mathrm{Rg} 1$ protects against acetaminophen-induced liver injury via activating Nrf2 signaling pathway in vivo and in vitro. Reg. Toxicol. Pharmacol. 98, 58-68. doi: 10.1016/j.yrtph.2018.07.012

Panieri, E., and Saso, L. (2019). Potential applications of NRF2 inhibitors in cancer therapy. Oxid. Med. Cell. Long. 9:193. doi: 10.3390/antiox9030193

Park, D.-J., Kang, J.-B., Shah, F.-A., Jin, Y.-B., and Koh, P.-O. (2020). Quercetin attenuates decrease of thioredoxin expression following focal cerebral ischemia and glutamate-induced neuronal cell damage. Neuroscience 428, 38-49. doi: 10.1016/j.neuroscience.2019.11.043

Ramsey, C. P., Glass, C. A., Montgomery, M. B., Lindl, K. A., Ritson, G. P., Chia, L. A., et al. (2007). Expression of Nrf2 in neurodegenerative diseases. J. Neuropathol. Exper. Neurol. 66, 75-85.

Reisman, S. A., Aleksunes, L. M., and Klaassen, C. D. (2009). Oleanolic acid activates $\mathrm{Nrf} 2$ and protects from acetaminophen hepatotoxicity via Nrf2-dependent and Nrf2-independent processes. Biochem. Pharmacol. 77, 1273-1282. doi: 10.1016/j.bcp.2008.12.028

Sachan, A. K., Das, D. R., and Kumar, M. (2016). Carum carvi-An important medicinal plant. J. Chem. Pharm. Res. 8, 529-533.

Shah, F.-A., Gim, S.-A., Kim, M.-O., and Koh, P.-O. (2014). Proteomic identification of proteins differentially expressed in response to resveratrol treatment in middle cerebral artery occlusion stroke model. J. Vet. Med. Sci. 76, 1367-1374. doi: 10.1292/jvms.14-0169

Shah, F. A., Gim, S. A., Sung, J. H., Jeon, S. J., Kim, M. O., and Koh, P. O. (2016). Identification of proteins regulated by curcumin in cerebral ischemia. J. Surg. Res. 201, 141-148. doi: 10.1016/j.jss.2015.10.025

Shah, F.-A., Kury, L. A., Li, T., Zeb, A., Koh, P. O., Liu, F., et al. (2019a). Polydatin attenuates neuronal loss via reducing neuroinflammation and oxidative stress in rat MCAO models. Front. Pharmacol. 10:663. doi: 10.3389/fnmol.2020.000663

Shah, F. A., Liu, G.-P., Kury, A., Tareq, L., Zeb, A., Abbas, M., et al. (2019b). Melatonin protects MCAO-induced neuronal loss via NR2A mediated prosurvival pathways. Front. Pharmacol. 10:297. doi: 10.3389/fnmol.2020. 00297

Shah, F. A., Zeb, A., Li, S., and Al Kury, L. T. (2019c). Pathological comparisons of the hippocampal changes in the transient and permeant middle cerebral artery occlusion rat models. Front. Neurol. 10:1178. doi: 10.3389/fnmol.2020.0001178

Shah, F.-A., Park, D.-J., and Koh, P.-O. (2018). Identification of proteins differentially expressed by quercetin treatment in a middle cerebral artery occlusion model: a proteomics approach. Neurochem. Res. 43, 1608-1623. doi: 10.1007/s11064-018-2576-x

Sivandzade, F., Bhalerao, A., and Cucullo, L. (2019). Cerebrovascular and neurological disorders: protective role of NRF2. Intern. J. Mol. Sci. 20:3433. doi: $10.3390 /$ ijms 20143433
Solleiro-Villavicencio, H., and Rivas-Arancibia, S. (2018). Effect of chronic oxidative stress on neuroinflammatory response mediated by CD $4+\mathrm{T}$ cells in neurodegenerative diseases. Front. Cell. Neurosci. 12:114. doi: 10.3389/fnmol. 2020.00114

Sung, J.-H., Shah, F.-A., Cho, E.-H., Gim, S.-A., Jeon, S.-J., Kim, K.-M., et al. (2012). Ginkgo biloba extract (EGb 761) prevents the ischemic brain injury-induced decrease in parvalbumin expression. Lab. Anim. Res. 28, 77-82.

Sung, J.-H., Shah, F.-A., Gim, S.-A., and Koh, P.-O. (2016). Identification of proteins in hyperglycemia and stroke animal models. J. Surg. Res. 200, 365-373. doi: 10.1016/j.jss.2015.07.020

Suzuki, T., and Yamamoto, M. (2015). Molecular basis of the Keap1-Nrf2 system. Free Rad. Biol. Med. 88, 93-100. doi: 10.1016/j.freeradbiomed.2015.06.006

Ullah, U., Badshah, H., Malik, Z., Uddin, Z., Alam, M., Sarwar, S., et al. (2020). Hepatoprotective effects of melatonin and celecoxib against ethanol-induced hepatotoxicity in rats. Immunopharmacol. Immunotoxicol. 42, 1-9.

Vila, N., Castillo, J., Dávalos, A., and Chamorro, A. (2000). Proinflammatory cytokines and early neurological worsening in ischemic stroke. Stroke 31, 2325-2329. doi: 10.1161/01.str.31.10.2325

Vomund, S., Schäfer, A., Parnham, M. J., Brüne, B., and Von Knethen, A. (2017). $\mathrm{Nrf2}$, the master regulator of anti-oxidative responses. Intern. J. Mol. Sci. 18:2772. doi: 10.3390/ijms18122772

Wang, Q., Tang, X. N., and Yenari, M. A. (2007). The inflammatory response in stroke. J. Neuroimmunol. 184, 53-68.

Wang, X. J., Hayes, J. D., Henderson, C. J., and Wolf, C. R. (2007). Identification of retinoic acid as an inhibitor of transcription factor Nrf2 through activation of retinoic acid receptor alpha. Proc. Natl. Acad. Sci. U.S.A. 104, 19589-19594. doi: 10.1073/pnas.0709483104

Winkel, A. F., Engel, C. K., Margerie, D., Kannt, A., Szillat, H., Glombik, H., et al. (2015). Characterization of RA839, a noncovalent small molecule binder to Keap1 and selective activator of Nrf2 signaling. J. Biol. Chem. 290, 28446-28455. doi: 10.1074/jbc.m115.678136

Ya, B.-L., Liu, Q., Li, H.-F., Cheng, H.-J., Yu, T., Chen, L., et al. (2018). Uric acid protects against focal cerebral ischemia/reperfusion-induced oxidative stress via activating Nrf2 and regulating neurotrophic factor expression. Oxid. Med. Cell. Long. 2018:6069150.

Yang, J., Su, J., Wan, F., Yang, N., Jiang, H., Fang, M., et al. (2017). Tissue kallikrein protects against ischemic stroke by suppressing TLR4/NF-кB and activating Nrf2 signaling pathway in rats. Exp. Therap. Med. 14, 1163-1170. doi: 10.3892/ etm.2017.4614

Yang, Q., Huang, Q., Hu, Z., and Tang, X. (2019). Potential neuroprotective treatment of stroke: targeting excitotoxicity, oxidative stress, and inflammation. Front. Neurosci. 13:1036. doi: 10.3389/fnmol.2020.0001036

Yeligar, S. M., Machida, K., and Kalra, V. K. (2010). Ethanol-induced HO1 and NQO1 are differentially regulated by HIF-1 $\alpha$ and Nrf2 to attenuate inflammatory cytokine expression. J. Biol. Chem. 285:35359. doi: 10.1074/jbc. $\mathrm{m} 110.138636$

Zhang, L., Wang, H., Zhou, Y., Zhu, Y., and Fei, M. (2018). Fisetin alleviates oxidative stress after traumatic brain injury via the Nrf2-ARE pathway. Neurochem. Intern. 118, 304-313. doi: 10.1016/j.neuint.2018.05.011

Zheng, J., Dai, Q., Han, K., Hong, W., Jia, D., Mo, Y., et al. (2020). JNK-IN-8, ac-Jun N-terminal kinase inhibitor, improves functional recovery through suppressing neuroinflammation in ischemic stroke. J. Cell. Physiol. 235, 2792-2799. doi: $10.1002 /$ jcp. 29183

Conflict of Interest: The authors declare that the research was conducted in the absence of any commercial or financial relationships that could be construed as a potential conflict of interest.

Copyright (c) 2020 Malik, Shah, Ali, Tan, Alattar, Ullah, Khan, Alshaman and Li. This is an open-access article distributed under the terms of the Creative Commons Attribution License (CC BY). The use, distribution or reproduction in other forums is permitted, provided the original author(s) and the copyright owner(s) are credited and that the original publication in this journal is cited, in accordance with accepted academic practice. No use, distribution or reproduction is permitted which does not comply with these terms. 